\title{
Article \\ Effect of the Coupled Pitch-Yaw Motion on the Unsteady Aerodynamic Performance and Structural Response of a Floating Offshore Wind Turbine
}

\author{
Ziwen Chen, Xiaodong Wang * and Shun Kang
}

Citation: Chen, Z.; Wang, X.; Kang, S. Effect of the Coupled Pitch-Yaw Motion on the Unsteady Aerodynamic Performance and Structural Response of a Floating Offshore Wind Turbine. Processes 2021, 9, 290. https://doi.org/ $10.3390 /$ pr9020290

Received: 21 December 2020

Accepted: 28 January 2021

Published: 3 February 2021

Publisher's Note: MDPI stays neutral with regard to jurisdictional claims in published maps and institutional affiliations.

Copyright: (c) 2021 by the authors. Licensee MDPI, Basel, Switzerland. This article is an open access article distributed under the terms and conditions of the Creative Commons Attribution (CC BY) license (https:// creativecommons.org/licenses/by/ $4.0 /)$.
Key Laboratory of Power Station Energy Transfer Conversion and System, Ministry of Education, North China Electric Power University, Beijing 102206, China; czwncepu@126.com (Z.C.);

kangs@ncepu.edu.cn (S.K.)

* Correspondence: wxd_ncepu@sina.com

\begin{abstract}
The floating offshore wind turbines (FOWTs) have many more advantages than the onshore wind turbines, but they also have more complicated aerodynamic characteristics due to complex platform motions. The research objective of this paper is to investigate unsteady aerodynamic characteristics of a FOWT under the pitch, yaw, and coupled pitch-yaw platform motions using the computational fluid dynamics (CFD) method in the Unsteady Reynolds Averaged Navier-Stokes (URANS) simulations. The pitch, yaw, and coupled pitch-yaw motions are studied separately to analyze the platform motions' effects on the rotor blade. The accuracy of the numerical simulation method is verified, and the overall performances, including power and thrust, are discussed. In addition, the comparison of total aerodynamic performance, force coefficients at different spans and structural dynamic response is provided. The numerical simulation results show that the platform pitching is the main influencing factor of power fluctuation, and the average thrust values of the pitch, yaw, and coupled motions are consistent regardless of the frequency value. The angle of attack (AOA) of airfoils is much more sensitive to the yaw motion, while the blade normal and tangential forces are mainly affected by pitch motion. As for the structural response, the results suggest that the aerodynamic loads of the wind turbine are more sensitive to the pitch motion, which is confirmed by the thrust force and torque of each blade during the platform motions.
\end{abstract}

Keywords: FOWT; platform pitching; platform yawing; aerodynamic performance; structural response

\section{Introduction}

Wind power has been developing rapidly as an important type of renewable energy. Wind energy has been being widely concerned in order to promote the energy source composition [1]. The installed capacity of wind power accounted for $28 \%$ the overall of renewable capacity additions by the end of 2018 [2-4]. As reported by the International Energy Outlook 2018, nearly 60\% of the new power generation capacity will be contributed by the year 2040, and $\mathrm{CO}_{2}$ emissions are expected to decrease to $1878 \mathrm{MT}[5,6]$.

In recent years, the installed capacity of onshore wind turbines has been markedly expanding but at a slower pace due to many affecting factors. A typical reason is that the aerodynamic noises caused by onshore wind turbines are not acceptable and may be worse than those from other environmental noise sources [7-9]. To achieve effective energy production, as well as to reduce capital costs [10], the size of wind turbine blades is growing significantly. Thus, large requirements for land areas may bring serious controversy with the existing used lands [11]. Therefore, there is a need for construction of wind farms in the deep sea (which is wider to generate electricity) in order to solve the problems caused by onshore wind turbines. In addition, with the increase in water depth, the flow distribution around the wind turbine becomes more complex, causing a tower shadow effect, wind 
shear, and so on [12]. A floating-base can reduce the complexity of flow distribution around a wind turbine and avoid the high instability of air-loads on wind turbines. Further research and development of floating offshore wind turbines (FOWTs) can be an effective solution to the above-mentioned problem.

Compared with onshore wind turbines, wind turbines built on a deep sea have many advantages. The wind in a deep sea is usually stronger and more uniform. Thus, by operating on a deep sea, a FOWT can make full use of wind resources and reduce noise pollution effectively. Under the combined influence of wind load and wave impact, the rotating plane of a FOWT is constrained in six degrees of freedom (6-DOF) direction [13], as shown in Figure 1. The three rotational components of the platform pitch around the $X$-axis, yaw around the $Y$-axis and roll around the $Z$-axis respectively due to wave and wind load. The three translational components are sway in the $x$-direction, heave in the $y$-direction, and surge in the $z$-direction. Bea [14] developed a numerical prediction method for fully coupled dynamic analysis of platform motions. The maximum accelerations are significantly increased due to the 6-DOF motions, which affects the corresponding inertial loading on blades. It can be seen that the aerodynamic performance and structural response are relevant to the 6-DOF motions. The coupled motion of the floating platform along with rotation bring significant variation to the aerodynamic and structural performance, which is the subject of this study, so as to provide a theoretical basis for design, operation, and maintenance of the FOWT.

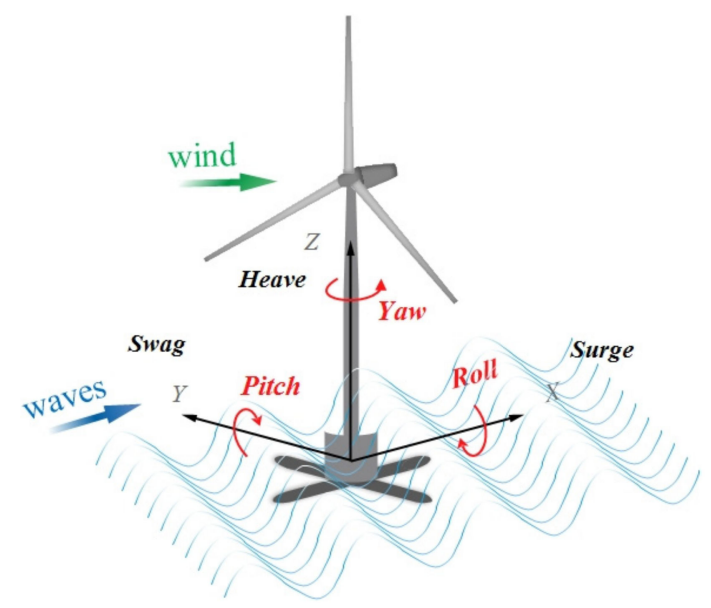

Figure 1. Motion types of the floating offshore wind turbine (FOWT).

A number of studies on aerodynamic load and structural modeling of the FOWT have been conducted. Moreover, the Blade Element Momentum (BEM) method was proposed. Tran and Kim [15] applied the unsteady blade element momentum theory and conducted the unsteady computational fluid dynamics simulation using the dynamic mesh technique to analyze the pitch motion of a wind turbine. They proposed an equivalent averaged velocity method that had a good consistency with the direct local relative velocity method. The generated wake strength behind the rotating blades during the forward pitch motion is stronger than that during the backward pitch motion. They also pointed out that the unsteady blade element momentum method had some theoretical limitations compared to the computational fluid dynamics (CFD) approach because of the large overall performance difference. In view of the limitation of the BEM, Qiu and Wang [16] proposed an unsteady numerical simulation method that uses a nonlinear lifting line method to compute the aerodynamic loads on the blades and a time-accurate free-vortex method (FVM) to simulate the wake. Compared to the BEM, the FVM can simulate the unsteady flow around the blade more accurately. Wen et al. $[17,18]$ analyzed the influences of the surge frequency and amplitude using the free vortex method. Their results showed that when the reduced frequency increased, the mean output power decreased at low tip speed ratios but increased 
at high tip speed ratios. In addition, the power performance of the FOWT under pitch motion was investigated using the FVM. The results showed that as frequency increased, the mean output power first decreased and then but increased. Hakjin Lee and Duck-Joo Lee [19] focused on studying unsteady wake characteristics of the FOWT. A nonlinear vortex lattice method (NVLM) was coupled with a vortex particle method (VPM) and used to study the translational and rotational motions of the wind turbine. The results show that the platform motions affected the wake evolution strongly and resulted in periodic deformation of the wake structure. As for the prediction of platform power and thrust, the vortex method usually provides satisfactory prediction. However, the aerodynamic performance of airfoils along the spanwise direction is not consistent with the prediction.

The limitation of vortex method promoted the application of experiments and CFD method. Hu [20] used a scaled wind turbine as a research object on a scale of 1:300, and the wind tunnel PIV (Particle Image Velocimetry) experiment was carried out to study the pitch motion with a platform amplitude of 5 degrees and a frequency of $0.3 \mathrm{~Hz}$. The near wake characteristics and aerodynamic loads of the wind turbine were studied. It was found that the change in the critical flow field was affected by the forward and backward motion of the wind turbine and was related to the incoming wind speed, amplitude, and frequency. Similarly, Bayati [21,22] carried out experiments on a 1/75 DTU (Technical University of Denmark) $10 \mathrm{MW}$ wind turbine and studied unsteady aerodynamics of the FOWT by using the wire anemometer and particle image velocimetry technology. This result provides an experimental reference for the operation of offshore wind farms.

Because of the difficulties of wind field experiments, especially for offshore wind power field, the limitation of space and equipment make the CFD method being a good choice, which can provide detailed results and substantial conclusions. Jun $\mathrm{Wu}$ [23] presented a method of unsteady CFD simulation of a floating offshore wind turbine that was based on the Reynolds Averaged Navier-Stokes (RANS) equations and the SST (Shear Stress Transfer) k-turbulence model. Liu [24] investigated the FOWT by coupling a highfidelity CFD solver with a general-purpose multi-body dynamics code. The impacts of blade flexibility and surge motion on wind turbine aerodynamics and structural responses were studied based on the CFD results of the flow field, force, and wake structure. Tran [25] investigated the NREL (National Renewable Energy Laboratory) 5 MW reference wind turbine under platform pitching in a prescribed sinusoidal function. An overset grid approach was used in Unsteady Reynolds Averaged Navier-Stokes (URANS) simulations to model the rigid body motion of a wind turbine blade. The CFD results were compared with the results of unsteady BEM and general dynamic wake (GDW) methods. For small amplitudes $\left(1^{\circ}-2^{\circ}\right)$ of the pitch motion, the results had a good agreement in general. However, the traditional methods showed over-predict assumptions when the oscillating amplitude increased. The rotating blades had interactions with their generated wake since the gap distance for blade-tip vortices decreased as the wind turbine moved downward and increased as the wind turbine moves forward [26].

Based on the literature survey, the platform motion of offshore wind turbine has been investigated to a certain degree. However, most of the studies analyzed the single motion of the platform pitching or yawing, while the coupled motion of the platform in several directions has been rarely reported. In addition, most of the research has been focused on wind turbines' overall aerodynamic performance. There has been less research on the aerodynamic force of each section of airfoils and the structural dynamic response of blades. Therefore, the main aim of this paper is to investigate the aerodynamic performance and structural response of the coupled motion, containing platform pitching and yawing. In the previous studies, unstructured mesh and dynamic mesh type methods were commonly used in the CFD simulations, which are usually difficult to perform. Wang $[27,28]$ proposed a simplified simulation method based on a multi-angular motion model and fully structured mesh in unsteady CFD simulations, which has been successfully used to simulate the dynamic yaw process of an onshore wind turbine. This paper uses the same model to investigate the unsteady aerodynamic characters of the FOWT under 
platform motions. In addition, this paper uses a fluid-solid coupling method to investigate the structural response of wind turbine blades during platform movement in order to make the investigation closer to reality. Furthermore, the coupled pitch-yaw motion is studied to observe the variation in unsteady aerodynamic performance.

The rest of the paper is organized as follows. Section 2 describes the geometry model and computational methods. Section 3 presents and discusses the verification results in detail. Section 4 draws the main conclusions gives future work directions.

\section{Floating Offshore Wind Turbine Model and Numerical Methods}

\subsection{Wind Turbine Model}

As a floating wind turbine, the NREL $5 \mathrm{MW}$ wind turbine is widely applied in the research, which is a three-bladed upwind wind turbine. In this turbine, the blade length is $63 \mathrm{~m}$ and hub is $90 \mathrm{~m}$ above the ground. The blade is constructed from NACA and DU series airfoils. In this study, three blades, hub, and a simplified nacelle are considered without the tower since the effect of the tower can be ignored [27]. The rated wind speed of the wind turbine is $11.4 \mathrm{~m} / \mathrm{s}$ with a rotational speed of $12.1 \mathrm{r} / \mathrm{min}$.

\subsection{Computational Domain and Boundary Conditions}

As shown in Figure 2, the computational domain is a rectangular zone that is made up of three main zones, the far-field zone, platform zone, and rotor zone. The external domain is a stationary zone that is $75 D$ in length and $50 D$ in width, where $D$ denotes the rotor diameter. The far field is used to set the inflow conditions, which belongs to the external domain, while the platform zone and rotor zone belong to the internal domain: (1) sphere platform zone with a radius of $7.5 \mathrm{D}$; (2) cylinder rotor zone with a $1.5 R$ radius and height of $12 \mathrm{~m}$. The platform zone is used to configure the pitch and yaw motions.

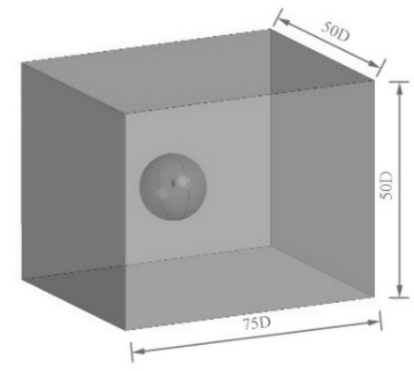

(a)

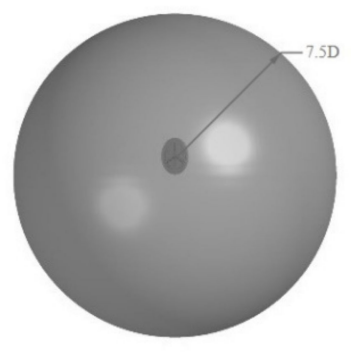

(b)

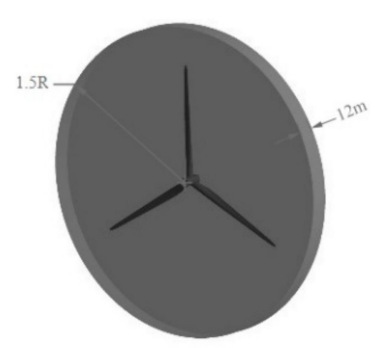

(c)

Figure 2. Computational zones: (a) far-field; (b) yaw zone; (c) rotational zone.

A uniform wind speed of $11.4 \mathrm{~m} / \mathrm{s}$, which is the rated wind speed, is set at the inlet of the domain with a turbulence intensity of $5 \%$. The up and bottom planes in the external domain are set as free-slip and no-slip walls, while the blade surface is set as a no-slip wall. The pressure outlet condition is assigned to the outlet of the domain. In the current case, the matching mesh interface is applied to the interface.

\subsection{Computational Mesh}

Fully structured mesh is employed for all domains. Multiple blocks are built for creating mesh since the relative rotation between zones can be investigated. The total number of girds is 10.31million. As shown in Figure 3, there is more than 3.96 million gird nodes for three blades using the NUMECA software (v11.2, NUMECA, Brussels, Belgium, 1992). The gird number for the far field is 5.27 million, which is enough to make the inflow through the internal domain. Platform sphere zone with 4.59 million grids is set to put the rotor in platform motion. In order to resolve the boundary layer, the grids around the 
blades were refined to keep $y+$ less than 5 on the blade surface, defined as follows, which satisfies the needs of the T (transition) -SST turbulence model.

$$
y^{+}=\frac{u^{*} y}{v}
$$

where $u^{*}$ is the friction velocity near the boundary, $y$ is the distance between the first layer grid node and the wall, and $v$ is kinematic viscosity of fluid.

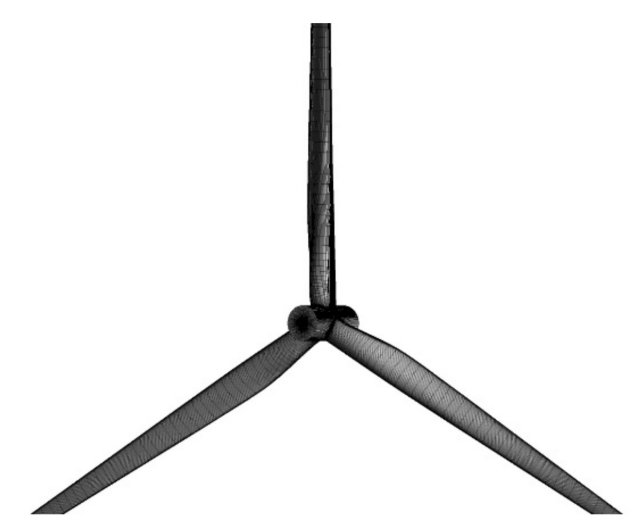

(a)

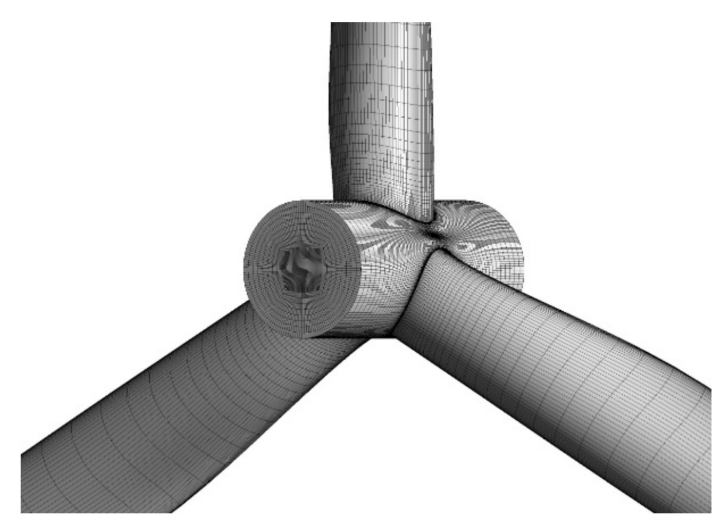

(b)

Figure 3. Computational mesh; (a) rotor; (b) blade surface.

\subsection{Numerical Methods}

The multi-angular motion model of an offshore wind turbine includes the motions of rotor rotation, platform pitching, platform yawing, and platform rolling. In this study, the effect of the platform's coupled pitch-yaw motion is investigated and compared with the individual motion of pitch or yaw. Both platform pitching and yawing adopt sinusoidal function as the law of motion. As shown in Figure 4, the variation in the platform angle is controlled by the angular velocity, which can be expressed as follows:

$$
\omega_{p}=2 \pi f A_{p} \cos (2 \pi f t),
$$

where $A_{p}$ denotes the pitching or yawing amplitude, and $f$ denotes the frequency. The cases with a uniform amplitude, $A_{p}=4^{\circ}$, and two different frequencies, $f=0.1 \mathrm{~Hz}$ and $f=0.2 \mathrm{~Hz}$ are investigated. The frequencies are chosen according to the most probable frequency of the waves at the rated wind speed [12].

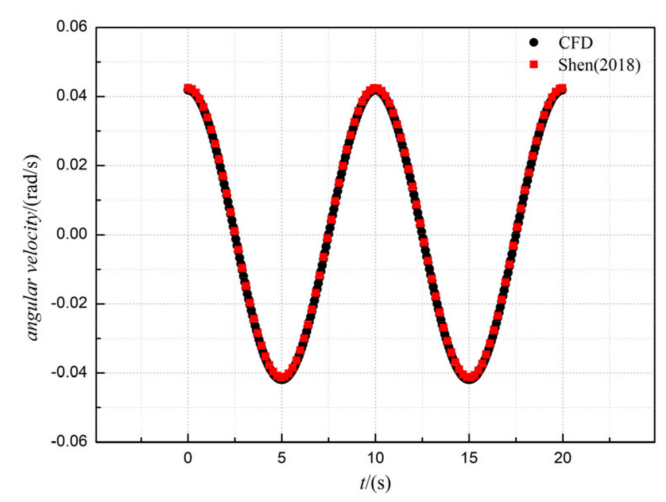

(a)

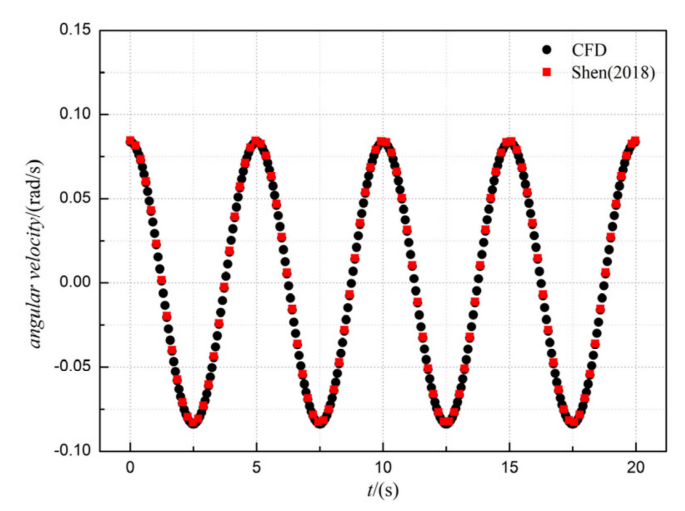

(b)

Figure 4. Angular velocity characteristics of dynamic motion, (a) $f=0.1 \mathrm{~Hz}$; (b) $f=0.2 \mathrm{~Hz}$. 
The maximum rotating speeds are $0.042 \mathrm{rad} / \mathrm{s}$ and $0.084 \mathrm{rad} / \mathrm{s}$. The maximum additional induced speeds of the platform pitch at the hub are $1 \mathrm{~m} / \mathrm{s}$ and $2 \mathrm{~m} / \mathrm{s}$, while the maximum additional induced speeds of the dynamic yaw process at the hub are $0.7 \mathrm{~m} / \mathrm{s}$ and $1.4 \mathrm{~m} / \mathrm{s}$. These speeds are distributed on the rotating blades at a certain height and phase. The induced velocity is superimposed with the incoming wind speed at different blade sections, which makes the output power and thrust of the wind turbine fluctuate.

Figure 5 shows the variations in the platform pitch and yaw angles at $f=0.1 \mathrm{~Hz}$. The negative degree indicates the reverse movement of the platform. For platform pitching, the wind turbine moves backward first. As shown in Figure 5, in the backward stage, the rotating plane of the wind turbine and vertical direction constitute the maximum positive angle at $t=1 / 4 T$. Then, in the forward stage, the wind turbine moves forward. The rotating plane of the wind turbine returns to the vertical direction at $1 / 2 T$. During the period from $1 / 2 T$ to $T$, the wind turbine makes a reverse movement. For platform yawing, the wind turbine carries out the positive yaw first; the yaw angle reaches $4^{\circ}$ at $1 / 4 T$. The rotation plane of the wind turbine returns to the yaw angle of zero at $1 / 2 T$ and carries out the adverse yaw. During the period from $1 / 2 T$ to $T$, the wind turbine conducts reverse deflection.

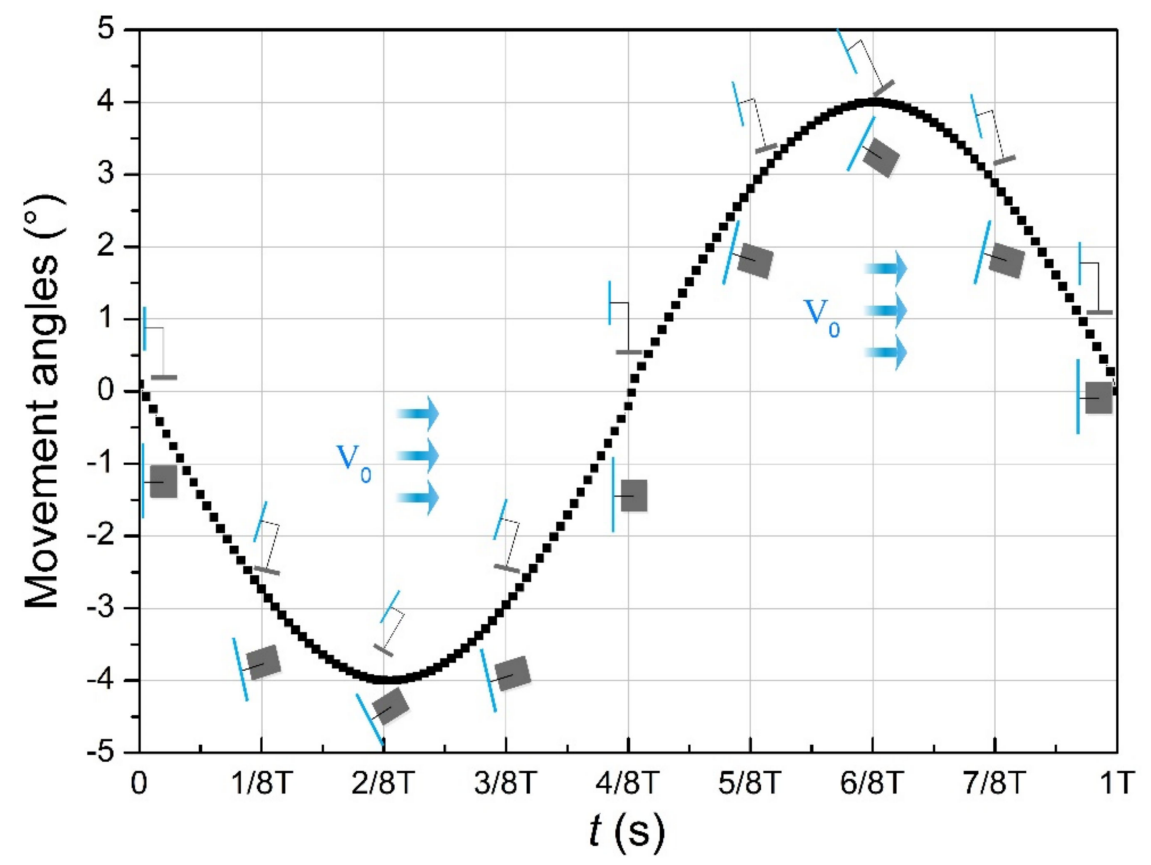

Figure 5. The variation of pitch and yaw angle at $f=0.1 \mathrm{~Hz}$.

The unsteady Reynolds-averaged Navier-Stokes equations (URANS) were solved in ANSYS Fluent. The commonly validated SIMPLE (Semi-Implicit Method for Pressure Linked Equations) method was used to solve the equations. The sliding mesh technique was used to exchange the data between the stationary zone and the rotational zone. As for turbulence modeling, the $k-\omega$ transitional SST turbulence model was used since it has been recognized as successful in simulating the offshore wind turbine by several studies on the CFD method $[13,27]$. A dual-time implicit time integration algorithm based on the second-order Euler backward differencing was used. The physical time-step size was $0.0342 \mathrm{~s}$, which was equivalent to a rotor azimuthal increment of $2.5^{\circ}$, and 20 pseudotime sub-iterations were used in each physical time step. Table 1 shows the sensitivity study of time-step size, which indicates that time-step size has no effect on the results in a certain scale. Considering the accuracy of unsteady simulation and time consumption, the mesoscale time step is selected. The residual of the continuity equation is reduced by more than four orders throughout the calculations. 
Table 1. Torque of different time-step sizes.

\begin{tabular}{ccccc}
\hline Time-step size $(\mathrm{s})$ & 0.01722 & 0.03423 & 0.06887 & Designed Value \\
\hline Torque $(\mathrm{N} \mathrm{m})$ & $4.07 \times 10^{6}$ & $4.07 \times 10^{6}$ & $4.07 \times 10^{6}$ & $4.08 \times 10^{6}$ \\
\hline Relative error & $0.24 \%$ & $0.24 \%$ & $0.24 \%$ & \\
\hline
\end{tabular}

The CFD method was used to simulate the platform pitching, platform yawing, and coupled pitch-yaw motions under two conditions $\left(A=4^{\circ}, f=0.1 \mathrm{~Hz}\right.$ and $\left.A=4^{\circ}, f=0.2 \mathrm{~Hz}\right)$, as shown in Table 2. The overall performance of the wind turbine was analyzed at $f=0.1 \mathrm{~Hz}$ and $f=0.2 \mathrm{~Hz}$. In addition, as for the flow details, under the condition of $f=0.1 \mathrm{~Hz}$, the variation in the aerodynamic coefficients along the blade and dynamic response load of the structure were considered to investigate the detailed unsteady aerodynamic performance of the FOWT. Next, the numerical method was verified, and the obtained results were analyzed. The sensitivity study of computational domain sizes and gird sizes are based on our previous research [13].

Table 2. Surge and pitch motion cases.

\begin{tabular}{cccc}
\hline Case & Motion & $\left.A_{p}{ }^{\circ}{ }^{\circ}\right)$ & $f(\mathbf{H z})$ \\
1 & Yaw & 4 & 0.1 \\
2 & Pitch & 4 & 0.2 \\
3 & Yaw and Pitch & 0.1 & 0.2 \\
4 & 4 & 0.1 \\
6 & & 4 & 0.2 \\
\hline
\end{tabular}

\section{Result Analysis}

\subsection{Verification Calculation Method}

The azimuth angle represents the position of the blade during clockwise rotation. The position of azimuth angle, $\varphi=45^{\circ}$, is shown in Figure 6 a. Figure $6 \mathrm{~b}$ illustrates the blade geometry angle of attack (AOA), represented by $\alpha$, shown on each airfoil section.

The pitch motion result is selected to verify the accuracy of the numerical method. The simulation results obtained by Shen [12] and Tran [25,26] are used for verification because there have been no experimental data available and consistent conditions. The variations in the wind turbine power coefficient $C_{p}$ is presented in Figure 7 , and this coefficient is defined as follows,

$$
C p=\frac{P}{\frac{1}{2} \rho A v^{3}},
$$

where $P$ denotes the output power of a wind turbine, $A$ denotes the rotation area, $\rho$ denotes the density of fluid, and $v$ denotes the incoming velocity. In the no-motion case, $C_{p}$ is constant, and in the other cases, it shows periodic variation. The generalized dynamic wake (GDW) method, free vortex method (FVM), and unsteady boundary method (UBEM) are compared with the CFD method in this paper. The peak value of $C_{p}$ in this work is slightly higher than that in the referenced results. This could be because the total turbulence model and the coarser mesh are applied to the model, which usually results in a rough-prediction of the output power. However, the periodicities of all results are nearly the same, which indicates that the simulations conducted in this work provide reasonable results. 


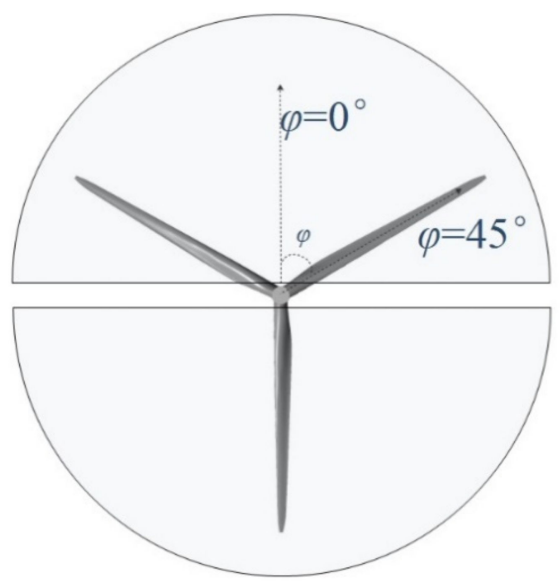

(a)

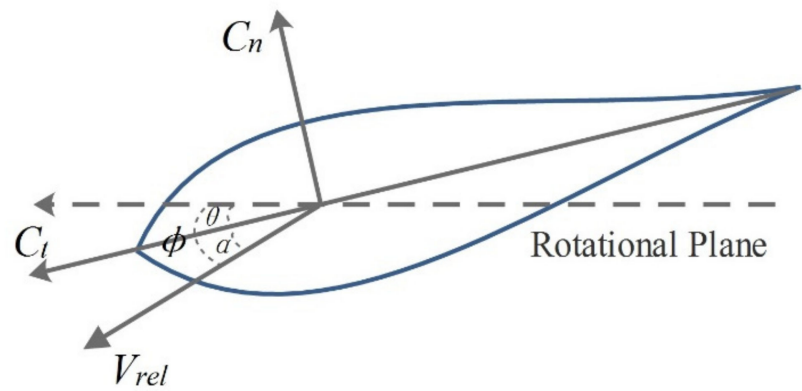

(b)

Figure 6. Schematic diagram of azimuth angle and angle of attack $(A O A)$. (a) Azimuth angle; (b) $A O A$.

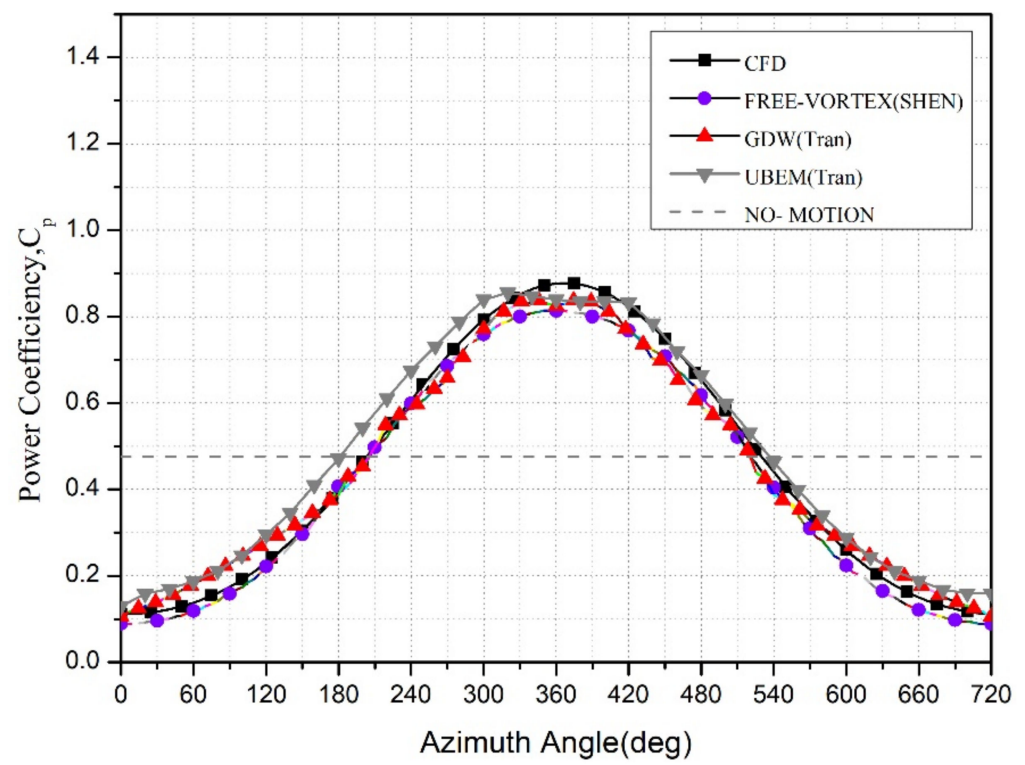

Figure 7. Power coefficient variation with the pitch motion at $A=4^{\circ}$ and $f=0.1 \mathrm{~Hz}$.

\subsection{Overall Performance Analysis}

The analysis of the overall aerodynamic performance mainly includes the output power and the whole force applied to FOWT. As shown in Figure 8, the overall performance of the wind turbine at $f=0.1 \mathrm{~Hz}$ and $f=0.2 \mathrm{~Hz}$ has consistent amplitudes. By comparing the platform motions of pitch, yaw, and coupled pitch-yaw, it can be noticed that the overall power and torque show fluctuation characteristics, and a higher frequency introduces 
larger aerodynamic fluctuations; also, a higher frequency corresponds to a larger power thrust fluctuation amplitude. In the case of the pitch motion, the maximum power of 11.7 MW, corresponding to $f=0.1 \mathrm{~Hz}$, is more than twice larger than the rated power. The maximum power corresponding to $f=0.2 \mathrm{~Hz}$ is even larger, reaching the value of $18.4 \mathrm{MW}$. In addition to the case of yaw motion, the maximum power of $5.5 \mathrm{MW}$ corresponding to $f=0.1 \mathrm{~Hz}$ is slightly lower than that corresponding to $f=0.2 \mathrm{~Hz}$, which has a value of 5.6 MW. Moreover, in the case of pitch-yaw motion, the maximum power corresponding to $f=0.1 \mathrm{~Hz}$ is $9.7 \mathrm{MW}$, and the maximum power corresponding to $f=0.2 \mathrm{~Hz}$ is $14.4 \mathrm{MW}$. As shown in Figure 8, the average power increases with the frequency. As for overall thrust, the increase in frequency will not have a significant impact on the average value of thrust but will increase the amplitude and fluctuation of the thrust.
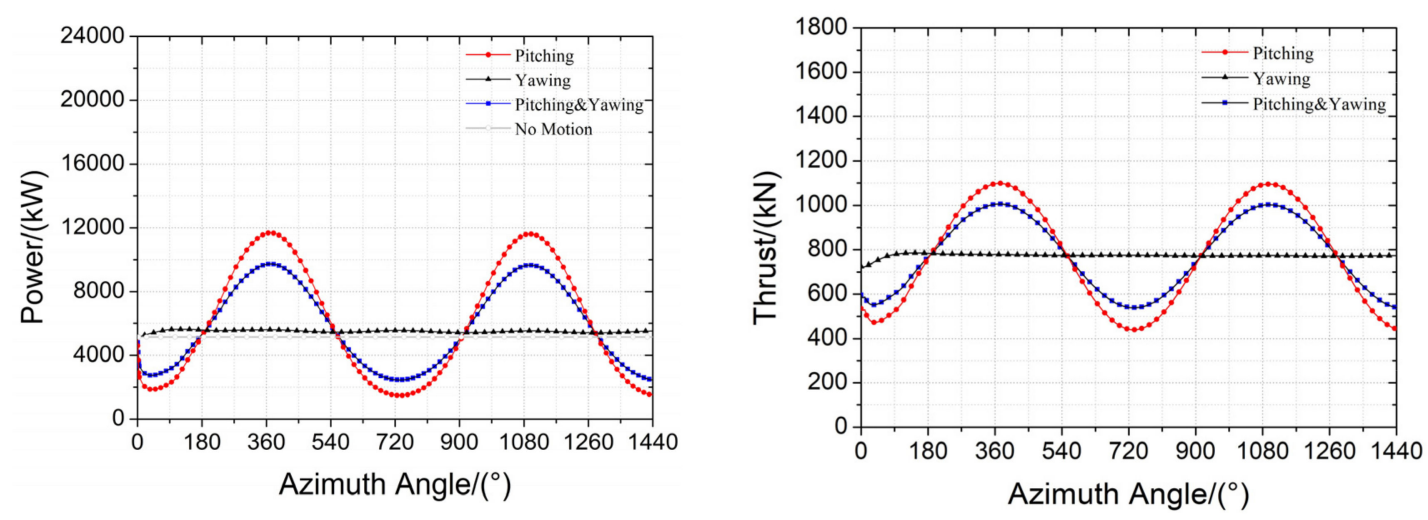

(a)
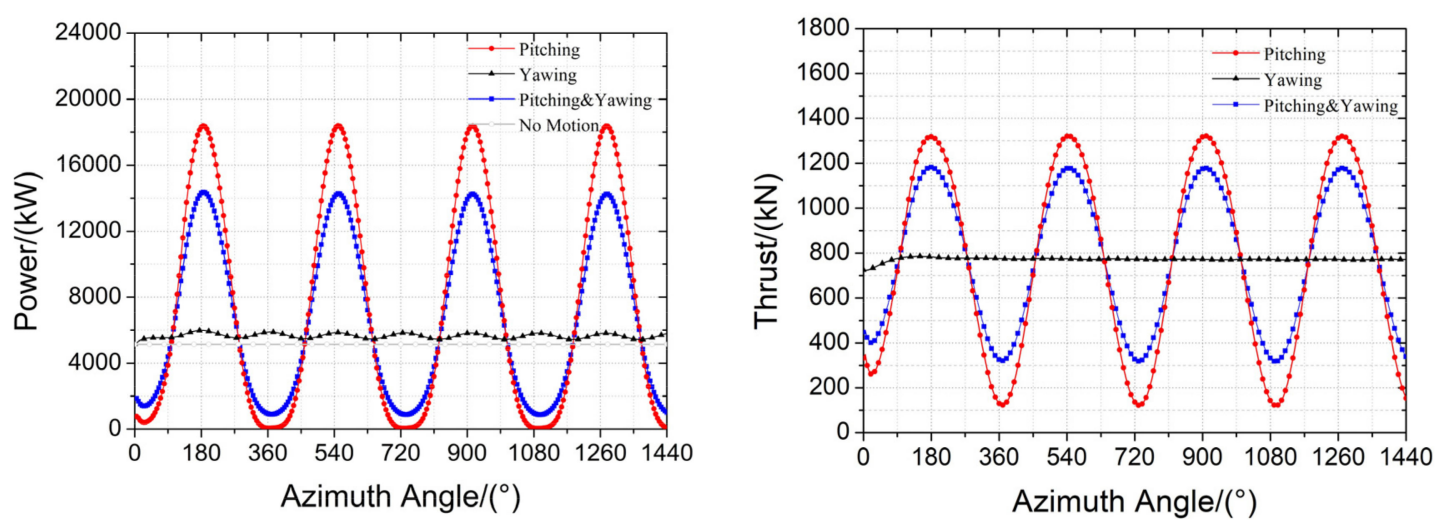

(b)

Figure 8. Variation in total performance. (a) $f=0.1 \mathrm{~Hz}$; (b) $f=0.2 \mathrm{~Hz}$.

Figure 9 shows that platform pitching is the main influencing factor of power fluctuation. This is due to the induced velocity generated by platform pitching. Furthermore, higher frequency corresponds to faster, induced velocity, which is the reason why the power fluctuation amplitude becomes larger at higher frequencies. Fluctuation in the power curve near the rated value is the effect of platform yawing on the output power. The reason is that the motion of platform yawing is a dynamic deflection process, which generates a slow induced velocity in the direction normal to the rotation plane. The induced velocity represents a product of the angular velocity of platform yawing and gyration radius, resulting in a sinusoidal fluctuation in the overall power. Compared to the pitch motion, the coupled motion increases the lower limit of the overall power but reduces its upper limit, which indicates that the coupled motion reduces the amplitude fluctuation of the power. However, as shown in Figure 9, coupled motion can reduce the average power generation. The reason is that the induced and incoming velocities are superposed in a 
positive direction when the platform makes a forward motion, and at the same time, the rotation plane has a certain offset angle due to platform yawing, which reduces the swept area of the rotor. The power amplitude is reduced because the output power is directly proportional to the third power of the wind velocity and swept area of the rotor. Yawing cannot significantly increase the averaged power generation regardless of frequency value. The thrust calculation does not consider the variation in the swept area, so the average thrust values of pitch, yaw, and coupled motions are basically consistent at $f=0.1 \mathrm{~Hz}$ and $f=0.2 \mathrm{~Hz}$.

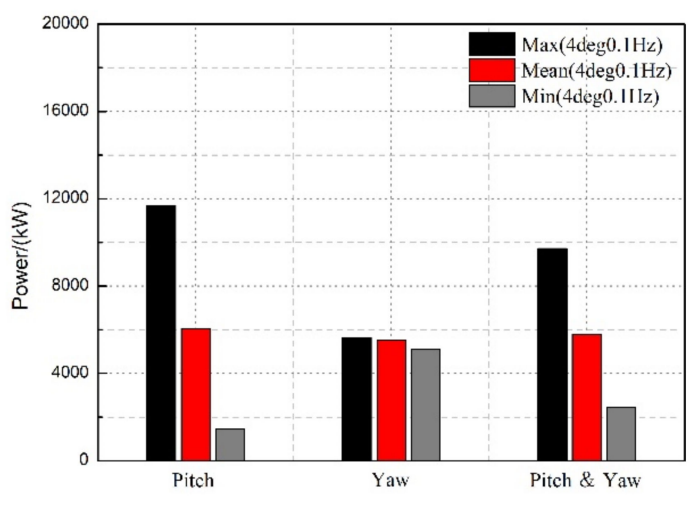

(a) $f=0.1 \mathrm{~Hz}$

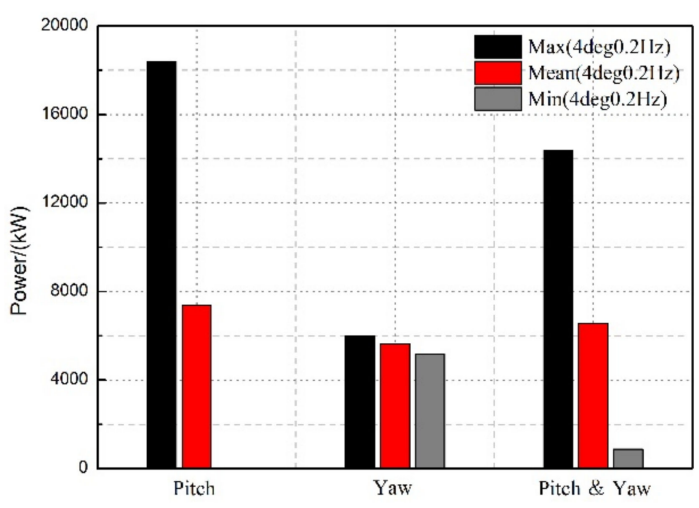

(c) $f=0.2 \mathrm{~Hz}$

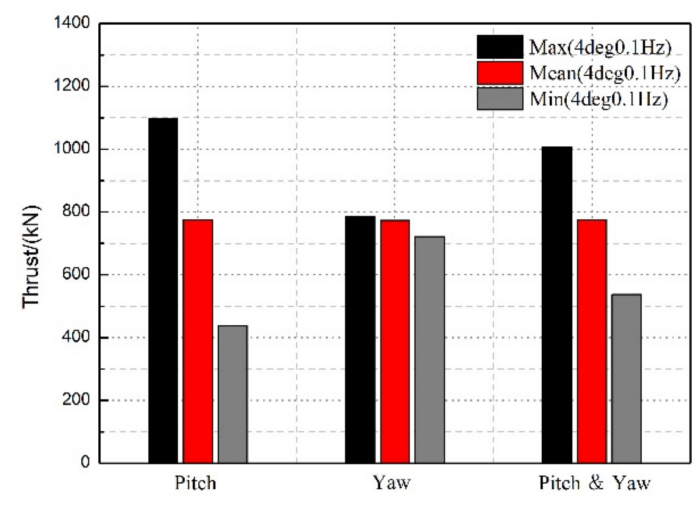

(b) $f=0.1 \mathrm{~Hz}$

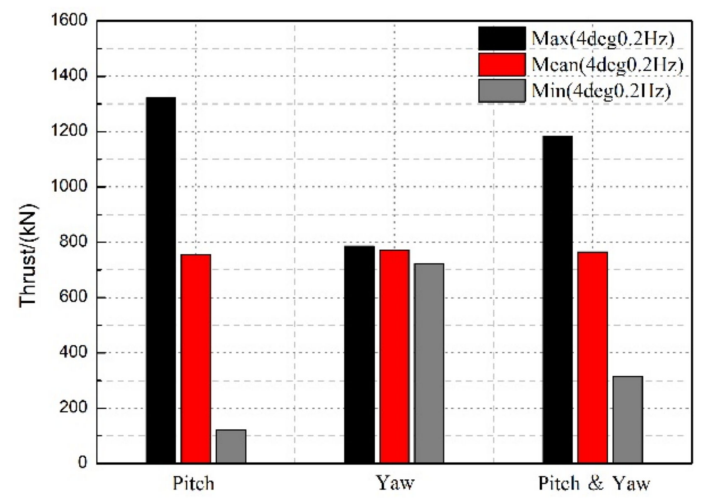

(d) $f=0.2 \mathrm{~Hz}$

Figure 9. Extreme and average values of the total performance.

\subsection{Aerodynamic Load Analysis along Spans of Blade}

Theory analysis is essential to determine the mechanism of platform motions. Figure 10 shows the velocity distribution during platform yawing, inducing an additional velocity $V_{d y n}$ that is normal to the rotation plane. The direction of $V_{d y n}$ is depended on the direction of yaw angular velocity. The yawing direction is determined by the right-hand rule, divided into two kinds of motion process:

(1) In the case of positive yawing angular velocity, $V_{d y n}$ has an acute angle with the direction of the incoming flow, which is consistent with the component of the inflow velocity in the vertical direction.

(2) In the case of negative yawing angular velocity, $V_{d y n}$ has an obtuse angle with the direction of the incoming flow, which is opposite to the component of the inflow velocity in the vertical direction.

$$
V_{\text {dyn_yaw }}=\left|\omega_{\text {yawing }} r \sin (\varphi)\right| \text {. }
$$


As shown in Figure 11, the velocity in the rotation plane can be projected into the radial and chordwise components denoted as $V_{r}$ and $V_{c} ; V_{r}$ has a radial three-dimensional effect on the blade, while $V_{c}$ causes the $A O A$ and sectional load to fluctuate periodically. The influence of $V_{c}$ can be calculated by:

$$
V_{\text {rel_yaw }}=\sqrt{\left(V_{o}(\cos \gamma-a)+\frac{\cos (2 \Pi f t)}{|\cos (2 \Pi f t)|} V_{\text {dyn_yaw }}\right)^{2}+\left(\omega r(1+b)-\frac{\sin (2 \Pi f t)}{|\sin (2 \Pi f t)|} V_{0} \sin \gamma \cos \varphi\right)^{2}} .
$$

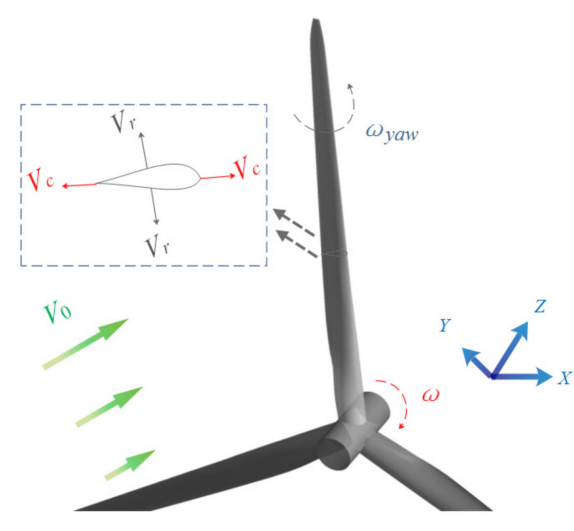

(a) Overall velocity distribution of rotor

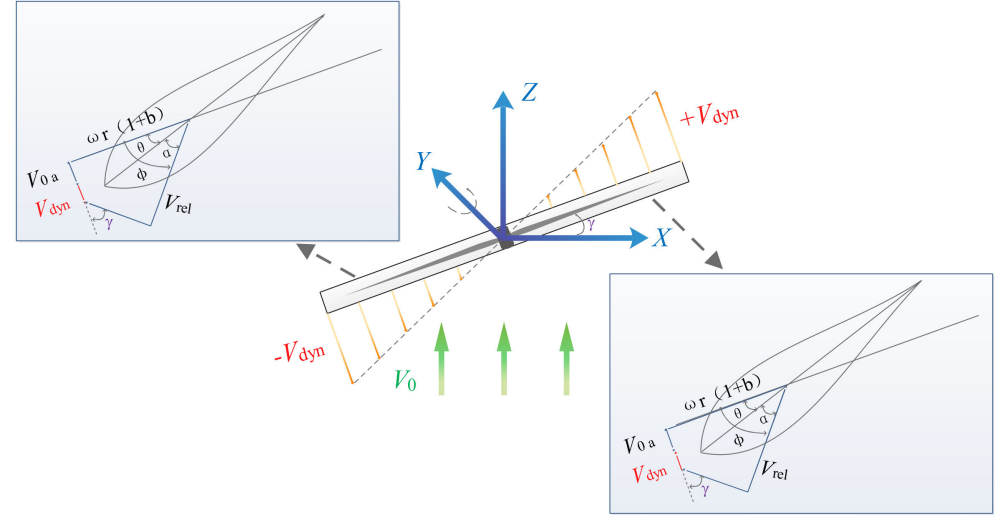

(b) Velocity distribution of airfoil section

Figure 10. Velocity diagram under yaw motion.

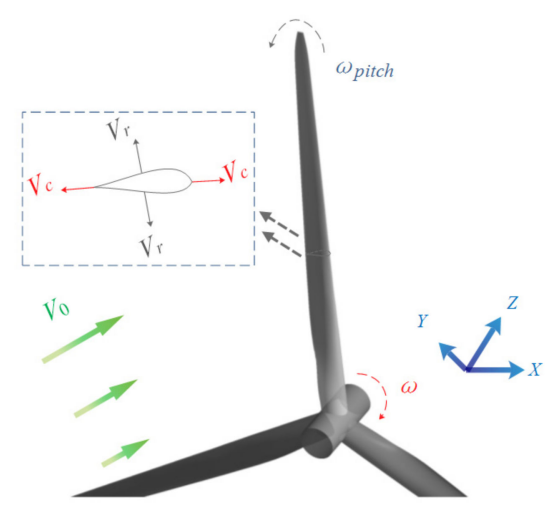

(a) Overall velocity distribution of rotor

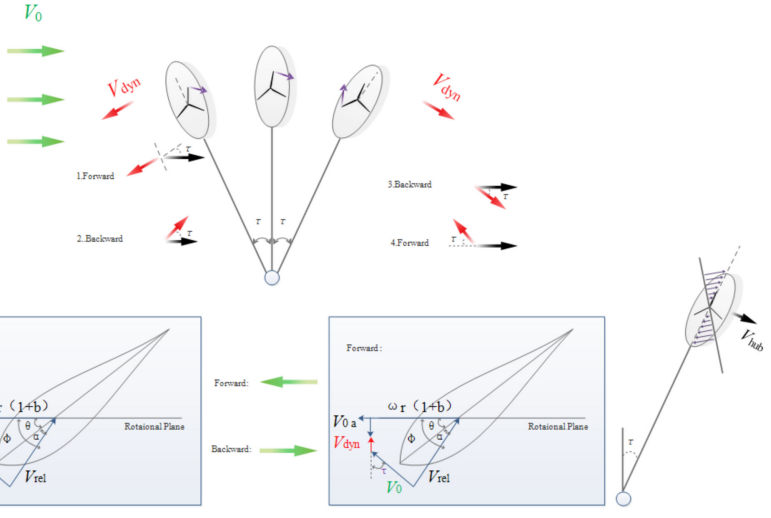

(b) Velocity distribution of airfoil section

Figure 11. Velocity diagram under pitch motion.

Platform pitching attaches an induced velocity defined as $V_{d y n}$ on the plane of rotation, which affects the aerodynamic performance of wind turbine. The aerodynamic performance of airfoils along the blade spanwise is analyzed in order to better explain the overall performance fluctuations. Figure 11 shows the velocity diagram of the airfoil along the blade spanwise. A velocity component on the rotation plane defined as $V_{\text {pitch }}$ is projected into the chordwise and radial components denoted $V_{c}$ and $V_{r}$. The direction of $V_{c}$ is defined by the angle of pitch motion. Besides, $V_{h u b}$ is wind speed at the hub, and $V_{d y n}$ is depended on the rotation plane position, and they are respectively expressed as:

$$
V_{h u b}=\frac{d \tau}{d t} H
$$




$$
V_{\text {dyn_pitch }}=\left(1+\frac{r}{R} \cos \varphi\right) .
$$

The movement can experience the following four states:

(1) The direction of $V_{d y n}$ is consistent with the inlet flow but opposite to the normal direction of the rotation plane as platform moving forward.

(2) The direction of $V_{d y n}$ is opposite to inlet flow but consistent with the normal direction of the rotation plane as platform moving backward.

(3) When the pitching angle is positive, $V_{\text {pitch }}$ direction is the positive $y$-axis direction, the rotation speed of the area above the hub plane is opposite to $V_{c}$, and the rotation speed of the area below the hub plane is the same as $V_{c}$.

(4) When the pitching angle is negative, $V_{\text {pitch }}$ direction is the negative direction of $Y$, the rotation speed of the area above the hub plane is the same as $V_{c}$, and the rotation speed of the area below the hub plane is the opposite to $V_{c}$.

The relative velocity $V_{\text {rel }}$ at the corresponding radius can be calculated by:

$$
V_{\text {rel_pitch }}=\sqrt{\left(V_{o}(\cos \tau-a)-\frac{\cos (2 \Pi f t)}{|\cos (2 \Pi f t)|} V_{\text {dyn_pitch }}\right)^{2}+\left(\omega r(1+b)-\frac{\sin (2 \Pi f t)}{|\sin (2 \Pi f t)|} V_{o} \sin \varphi \sin \tau\right)^{2}}
$$

In addition, based on the above analyses, the relative velocity of the coupled pitch-yaw motion can be calculated by:

$$
V_{\text {rel }}=\sqrt{\left(V_{o}(\cos \tau-a)-\frac{\cos (2 \Pi f t)}{|\cos (2 \Pi f t)|} V_{\text {dyn_pitch }}+\frac{\cos (2 \Pi f t)}{|\cos (2 \Pi f t)|} V_{\text {dyn_yaro }}\right)^{2}+\left(\omega r(1+b)-\frac{\sin (2 \Pi f t)}{|\sin (2 \Pi f t)|} V_{o} \sin \varphi \sin \tau-\frac{\sin (2 \Pi f t)}{|\sin (2 \Pi f t)|} V_{0} \sin \gamma \cos \varphi\right)^{2}}
$$

The $A O A$ distribution in three typical spanwise directions is presented in Figure 12. Platform yawing and coupled motion have consistent regular patterns in terms of $A O A$ variation, which indicates the yawing motion has a major impact on the variation in $A O A$. Coupled pitch-yaw motion makes $A O A$ fluctuate more violently in the case of the forward pitching and positive yawing. However, in the case of backward pitching and negative yawing, the coupled motion makes $A O A$ fluctuate more smoothly.

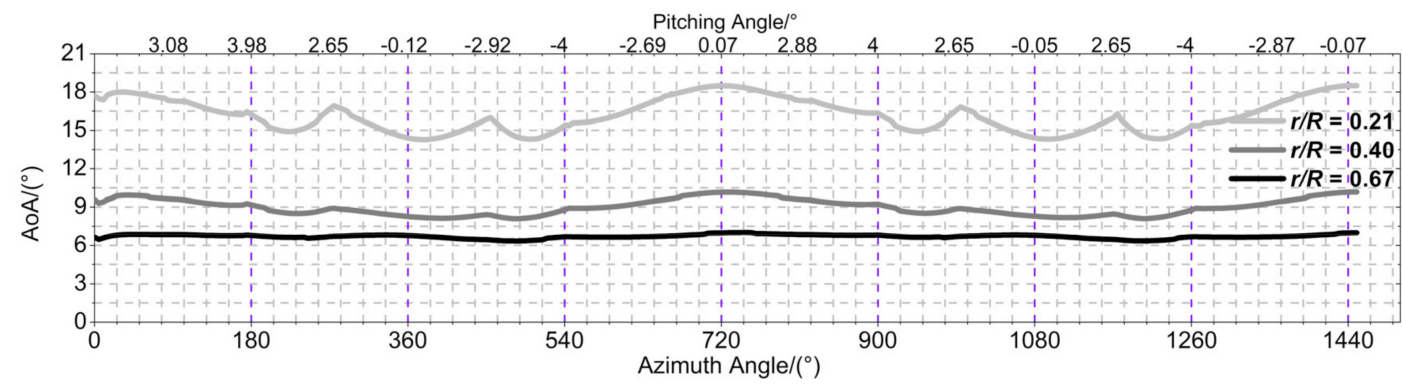

(a)

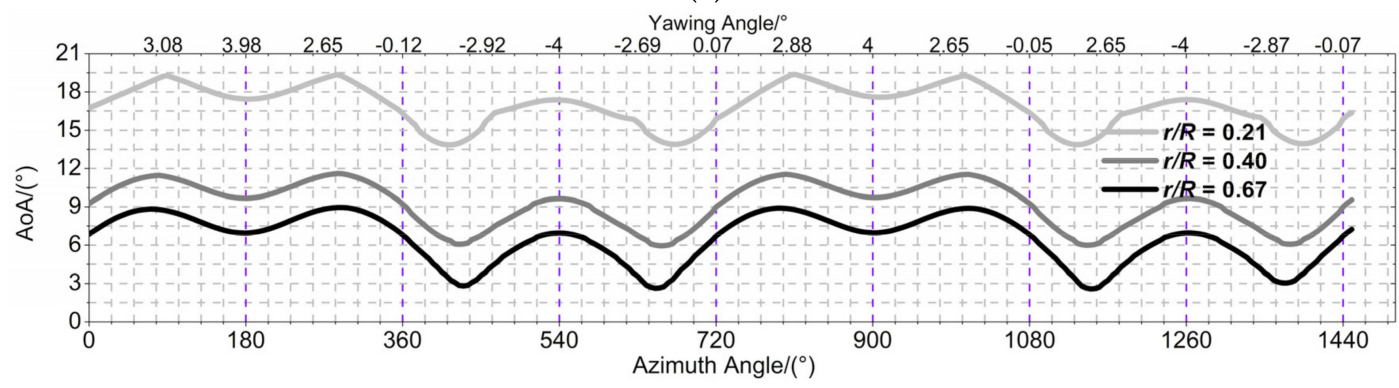

(b)

Figure 12. Cont. 


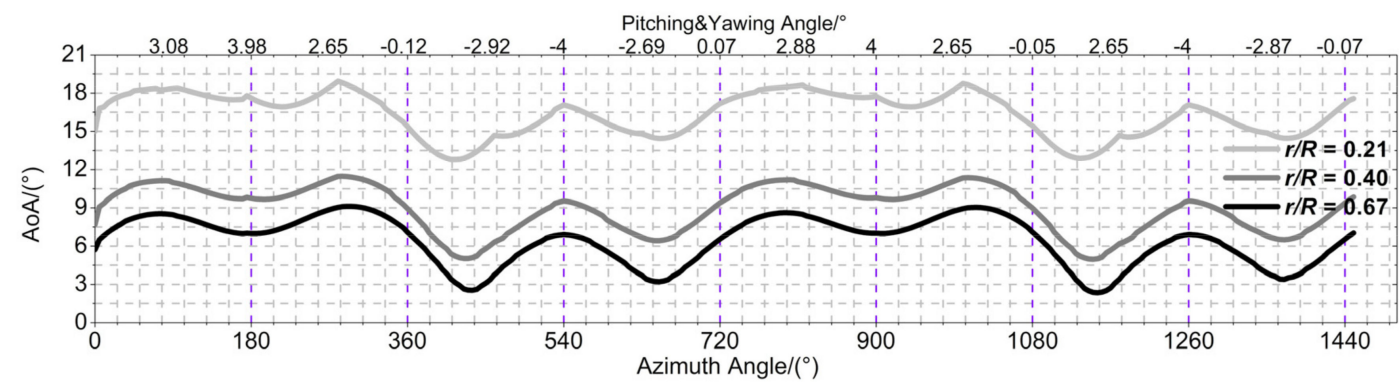

(c)

Figure 12. Variation in $A O A$ at different spans of platform motions. (a) pitch motion; (b) yaw motion; (c) coupled pitch-yaw motion.

The variations in the aerodynamic load along the blade direction are presented in Figures 13 and 14. For platform motions, a modest fluctuation in the aerodynamic loads can be observed. Figure 13 shows the variation of the normal force coefficients at three sections $(r / R=0.21,0.4,0.67)$ in two periods, the normal force coefficient of dimensionless parameter is defined as, which is shown in Figure $6 b$,

$$
C_{n}=\frac{F_{n}}{2 \rho A V_{r e l}^{2}} .
$$

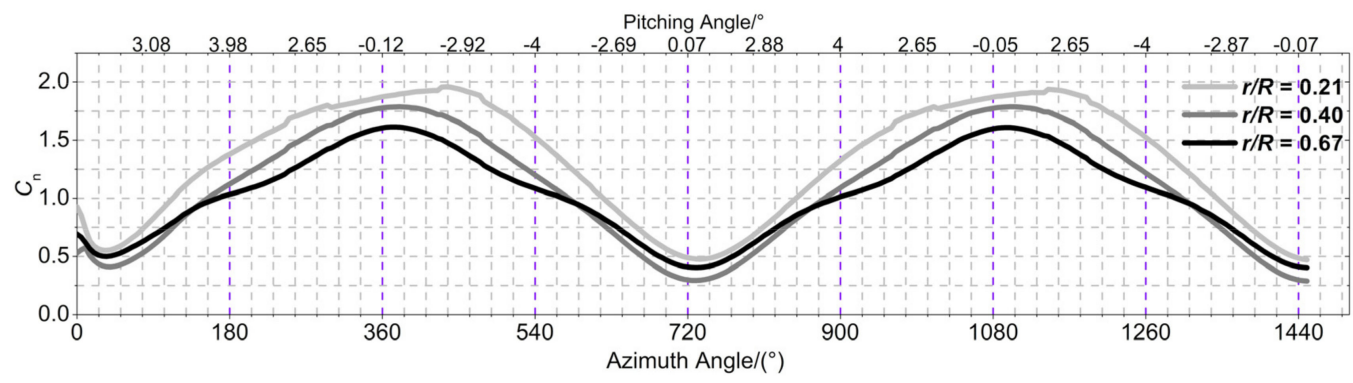

(a)

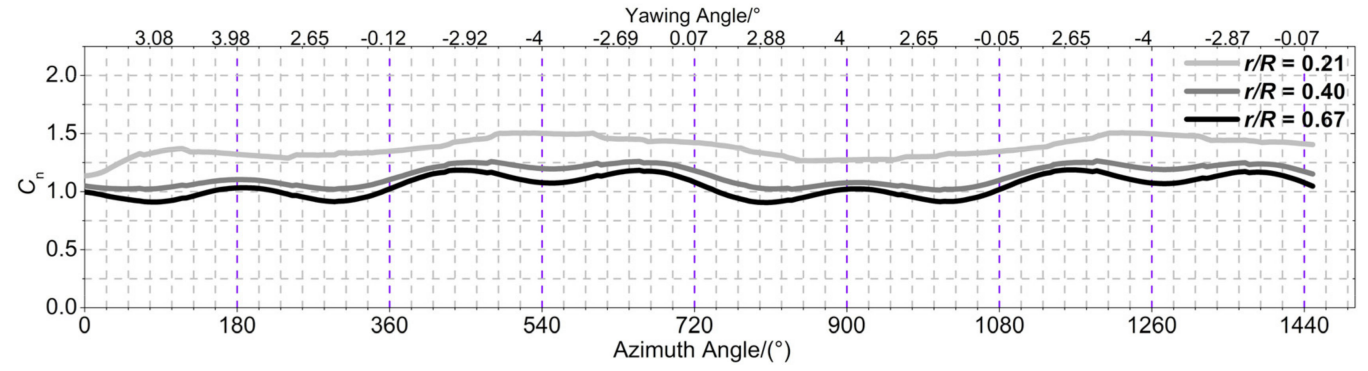

(b)

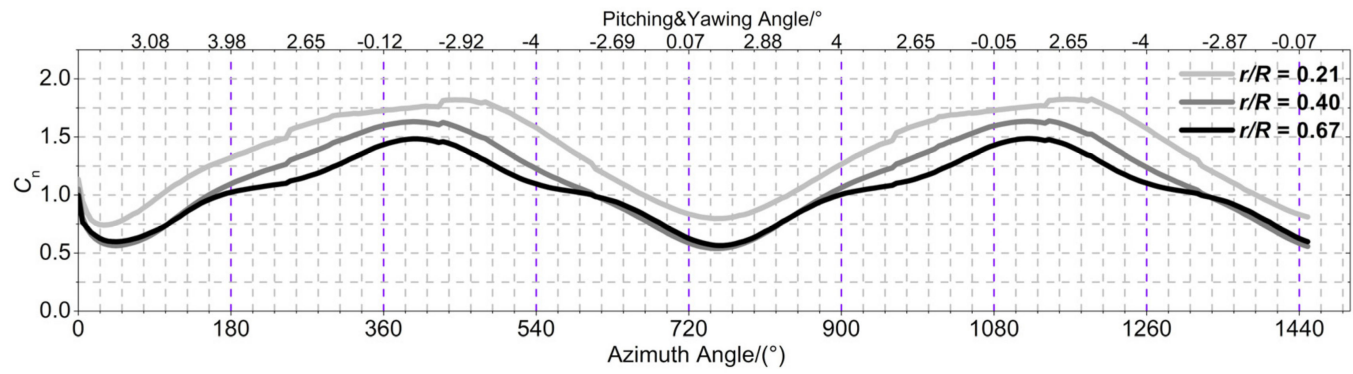

(c)

Figure 13. Variation in normal force coefficient at different spans for the platform motions. (a) pitch motion; (b) yaw motion; (c) coupled pitch-yaw motion. 


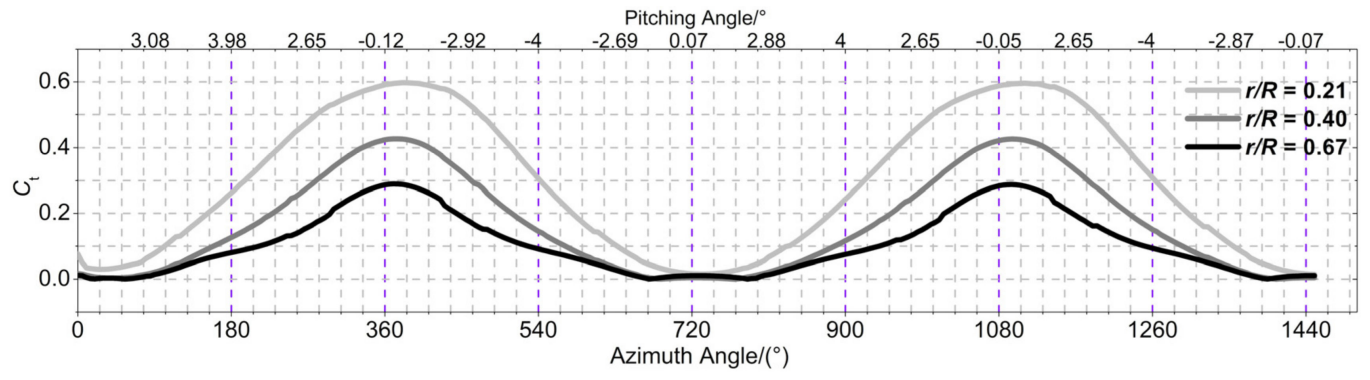

(a)

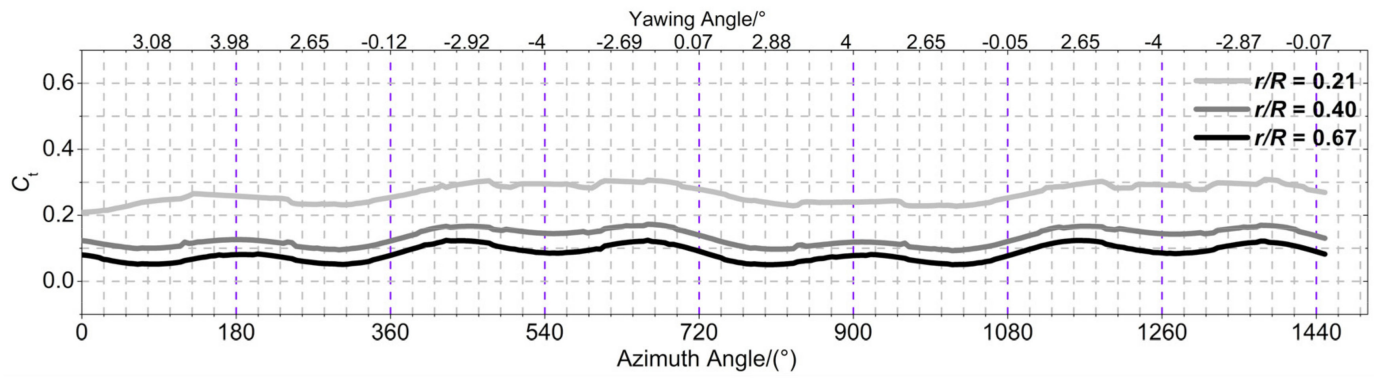

(b)

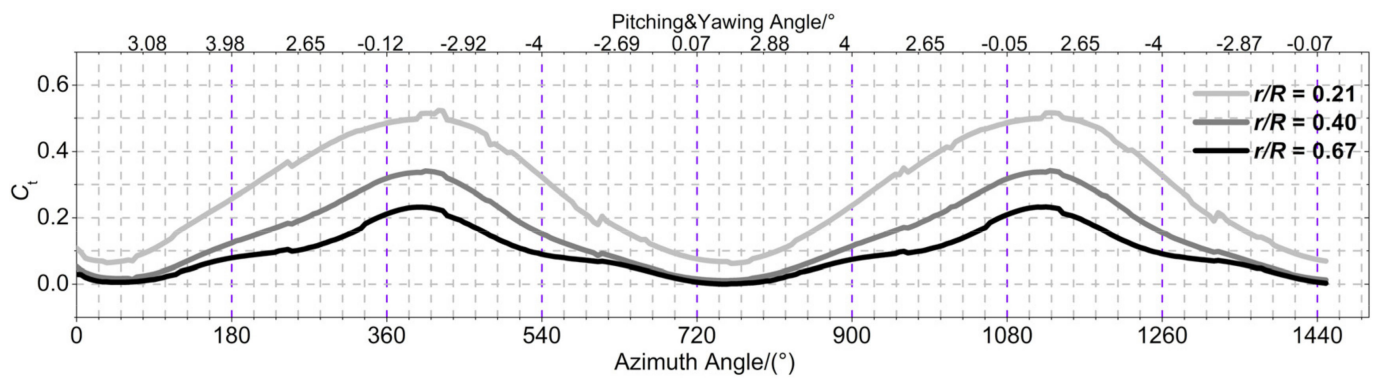

(c)

Figure 14. Variation in tangential force coefficient at different spans for the platform motions. (a) pitch motion; (b) yaw motion; (c) coupled pitch-yaw motion.

As shown in Figure 8, the variation in the normal force coefficient is consistent with that of the overall power. At the amplitude of $4^{\circ}$, the normal force coefficients of sections $r / R=0.4$ and $r / R=0.67$ are smaller than that of section $r / R=0.21$. By comparing the three types of platform motions, it can be found that the platform pitching and coupled motions have the same variations in the normal force coefficient, which shows the blade normal force is mainly affected by the pitch motion. The maximum normal loads of the pitch motion and coupled motions appear at the azimuth of $360^{\circ}$ and $1080^{\circ}$, respectively, while the minimum value appears at the azimuth of $720^{\circ}$. However, the fluctuation amplitude of the normal force coefficient of the coupled motion is smaller than that of the pitch motion. Near the azimuth angle of $720^{\circ}$, for the platform pitching, the normal force on the outer spanwise blade is larger than that on the middle spanwise blade, but the normal force on the outer spanwise blade is the same as that on the middle spanwise blade. The reason is that the normal load of the outer blade spread near the azimuth angle of $720^{\circ}$ in the yaw motion is smaller than that of the middle blade spread, as shown in Figure 13. This indicates that the normal force of the blade is weakened by the participation of yaw motion. The distribution of the tangential force coefficients at three sections in two periods is shown in Figure 14. The variation in the tangential force coefficient relative to the azimuth angle 
is consistent with the change in the output power. The tangential force coefficient of dimensionless parameter shown in Figure $6 \mathrm{~b}$ is defined as,

$$
C_{t}=\frac{F_{t}}{2 \rho A V_{r e l}^{2}} .
$$

The positions where the maximum and minimum values appear are the same as those of the normal force coefficient, but the amplitude of variation is obviously larger than that of the normal force coefficient. Thus, the tangential force coefficient is closely related to the output of the torque and power, which explains why the power fluctuation of the coupled motion in Figure 8 is smaller than that of the pitch motion. By comparing $C_{t}$ of the three motions, it can be found that variations in $C_{t}$ are consistent for pitch and coupled motions. This indicates that $C_{t}$ is mainly affected by the pitch motion, while the yaw motion has a smaller influence on the tangential force of the wind turbine blade. Specifically, the constant curve of the tangential force coefficient appears near the azimuth angle of $720^{\circ}$. The reason is that the component in the tangential direction of the induced velocity, caused by the pitch motion, is opposite to the direction of the incoming velocity, which decreases the tangential force smaller and makes $C_{t}$ more stable. Therefore, the torque and output power also reach the minimum values, as shown in Figure 8. Due to the influence of the yawing induced-velocity, the coupled motion fluctuates near the azimuth angle of $720^{\circ}$, but the tangential load of the inner span is still larger than that of the middle span and outer span, which is also the reason why the minimum output power of the coupled motion is larger than that of the pitch motion.

\subsection{Wake Flow Characteristics}

The flow analysis is essential in studying the characteristics of the flow field as well as the effects of different motion types. In Figure 15, there is a comparison of the wake flows induced by the platform motions, and they are divided into four positions, in the YZ plane. The distinctions of wake field between pitch, yaw, and coupled pitch-yaw motions are analyzed at $A=4^{\circ}, f=0.1 \mathrm{~Hz}$.

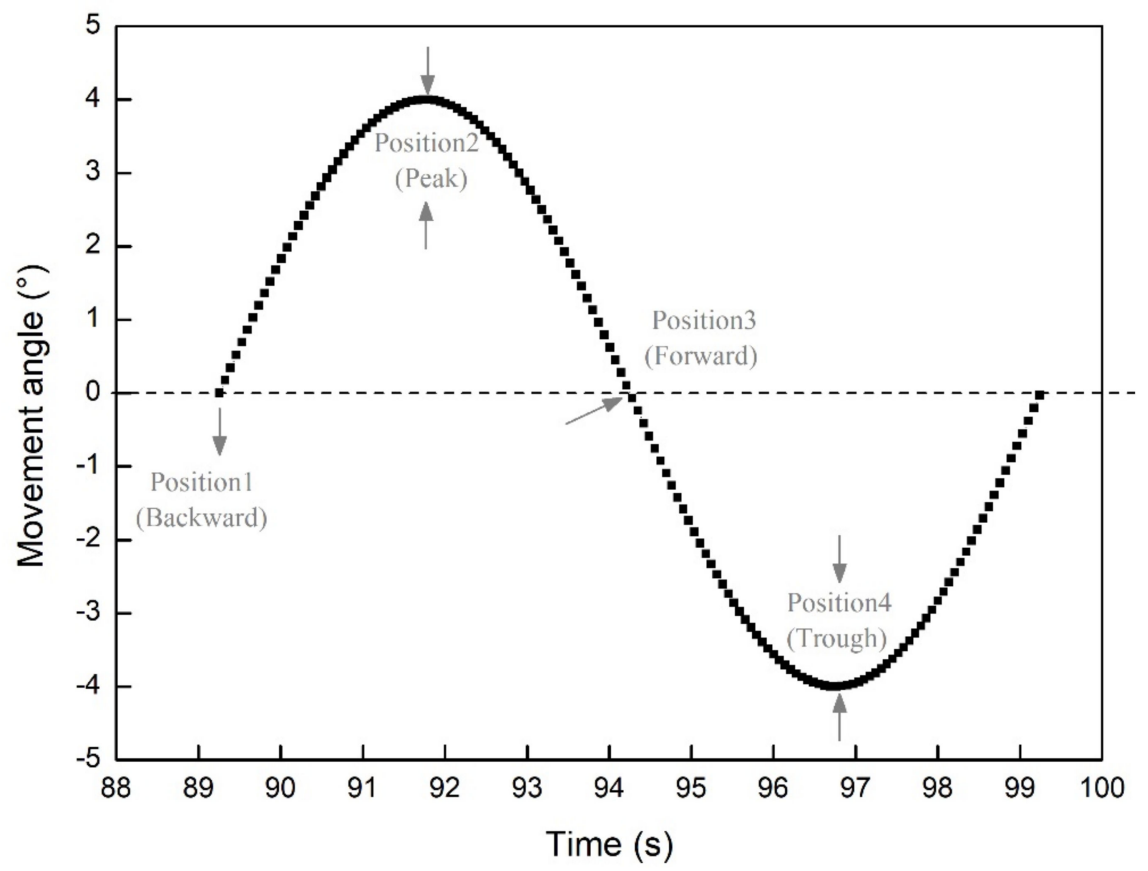

Figure 15. The different positions during the process of movement. 
(1) Position 1. At the initiation of the movement, as shown in Figure 16, the wake regions showed a certain level of symmetry, but the pitch and yaw motions acted differently in the wake distribution. The pitch motion induced a larger velocity distribution near the hub. The wake of the coupled motion also caused a high-velocity distribution because of the pitch motion, while the yaw motion could reduce the induced velocity behind the hub.

(2) Position 2. Figure 17 shows the velocity contours at the position of the maximum movement position. Compared to the initial position, the wake deflection occurred, and the symmetry of the contours was significantly reduced. In addition, the near wake region of the platform pitching produced a larger-velocity region compared to the yawing. Moreover, the participation of yaw motion reduces the velocity near hub in the coupled pitch-yaw motion, and reduces the flowing complexity in the tip vortex system.

(3) Position 3. Figure 18 illustrates the z-velocity distribution at the forward movement, which included the most violent motion due to the maximal induced velocities. The near wake flow presented the consistent state for the different motions. The coupled pitch-yaw motion produced a similar blade tip vortex structure as the pitch motion indicating that there is a stronger effect of the pitch motion on the forward stage.

(4) Position 4 . The maximum amplitude, i.e., the amplitude of $4^{\circ}$, the velocity gradient near the hub region was quite large, especially for the coupled pitch-yaw motion, shown in Figure 19. Compared with the position 1 of the rotor, on the contrary, the participation of yaw motion increases the velocity near hub in the coupled pitch-yaw motion. However, at position 4, the vortex structure of the single pitch motion and the coupled pitch-yaw motion is almost the same at the blade tip.

Velocity in Stn Frame

$\left[\mathrm{m} \mathrm{s}^{-1}\right]$

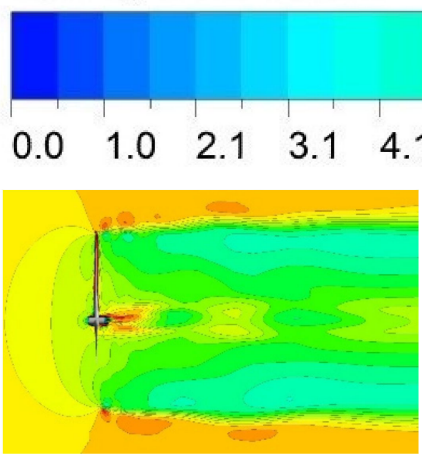

Pitch

\section{$\begin{array}{llllllllll}4.1 & 5.2 & 6.2 & 7.3 & 8.3 & 9.3 & 10.4 & 11.4 & 12.4 & 13.5\end{array}$}

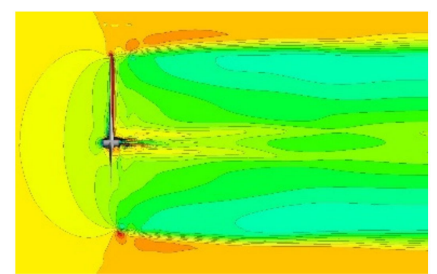

Yaw

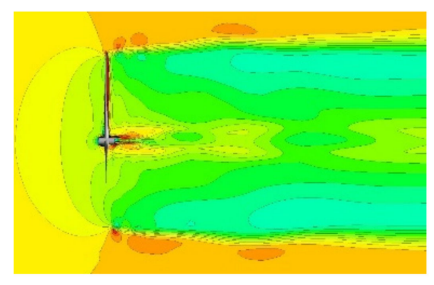

Pitch-yaw

Figure 16. Velocity distribution at Position 1.

Velocity in Stn Frame

$\left[\mathrm{m} \mathrm{s}^{-1}\right]$

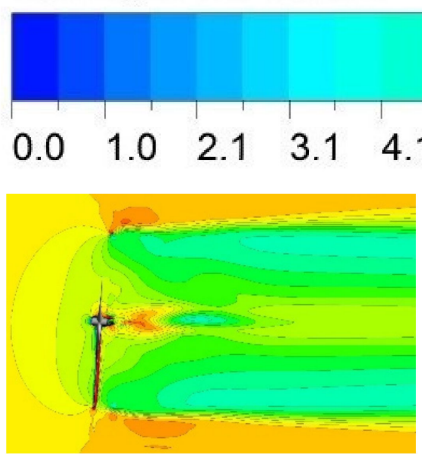

Pitch

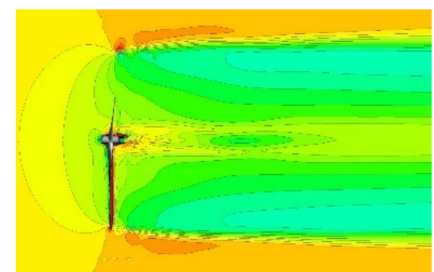

Yaw
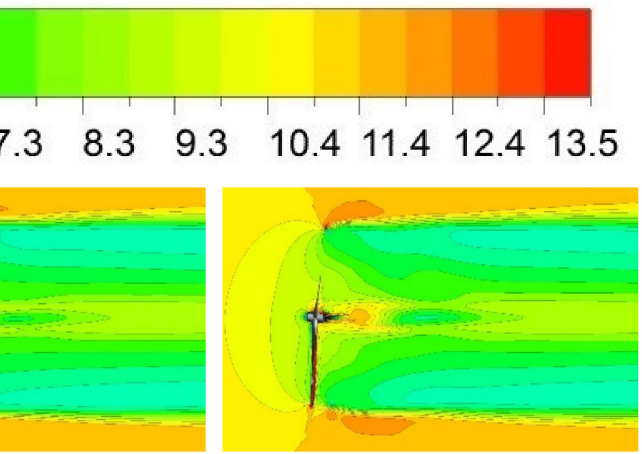

Pitch-yaw

Figure 17. Velocity distribution at Position 2. 
Velocity in Stn Frame $\quad\left[\mathrm{m} \mathrm{s}^{-1}\right]$

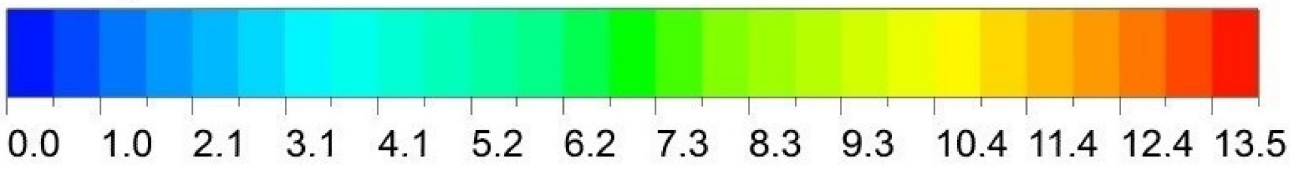

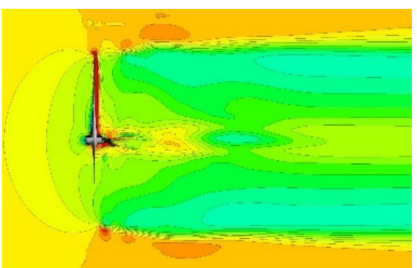

Pitch

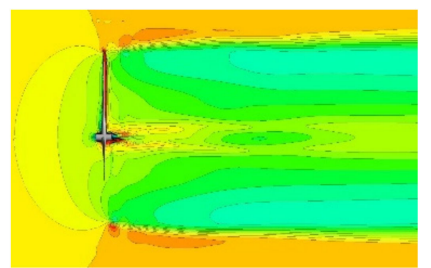

Yaw

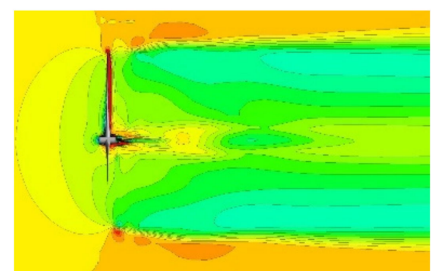

Pitch-yaw

Figure 18. Velocity distribution at Position 3.

Velocity in Stn Frame

$\left[\mathrm{m} \mathrm{s}^{-1}\right]$

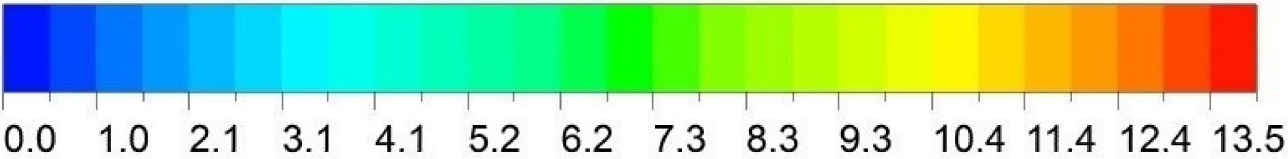

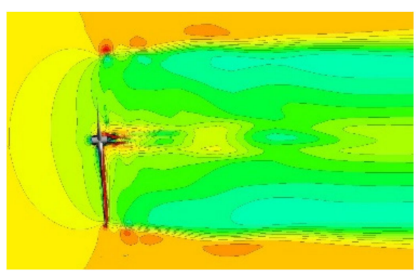

Pitch

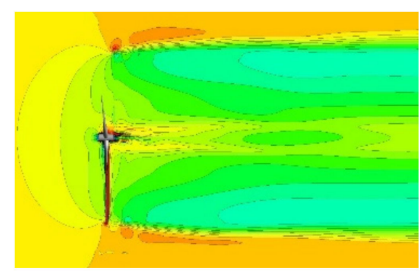

Yaw

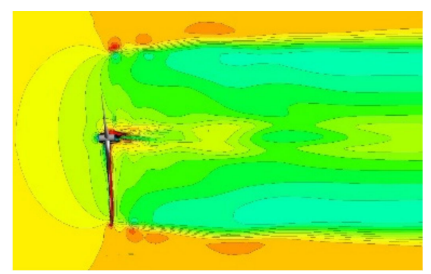

Pitch-yaw

Figure 19. Velocity distribution at Position 4.

\subsection{Structural Load Analysis}

During the operation of FOWT, the blades can deform due to the combination of wind load and waves, among which the deformations in flapwise and edgewise direction are the most significant. After the aerodynamic load analysis, the pre-stressed response of the blade can provide a theoretical basis of the FOWT structure construction. For the purpose of studying the influence of platform motions on the blade structure, the finite element model was established based on the stiffness and mass distribution along the blade, which was divided into several sections. The built finite element model is presented in Figure 20. The elastic modulus and density of each section were calculated. As shown in Figure 21, the blade shell structure was meshed, and the number of that was about 49,000 . The modal analysis results are presented in Table 3, where it can be seen that they coincide well with the results calculated by Fatigue Aerodynamic Structure Turbulence (FAST) and Automatic Dynamic Analysis of Mechanical Systems (Adams), which are two kinds of analysis software (v12.0, MSC, US, 2002) for multibody structure dynamics. $\triangle$ is the relative deviation between the results of mechanical method and those of the other two methods. The structural response comparison is analyzed at an amplitude of $A=4^{\circ}$ and a frequency of $f=0.1 \mathrm{~Hz}$. The results verify the accuracy of blade shell modeling.

$$
\triangle=\left|f-f^{\prime}\right| / f
$$




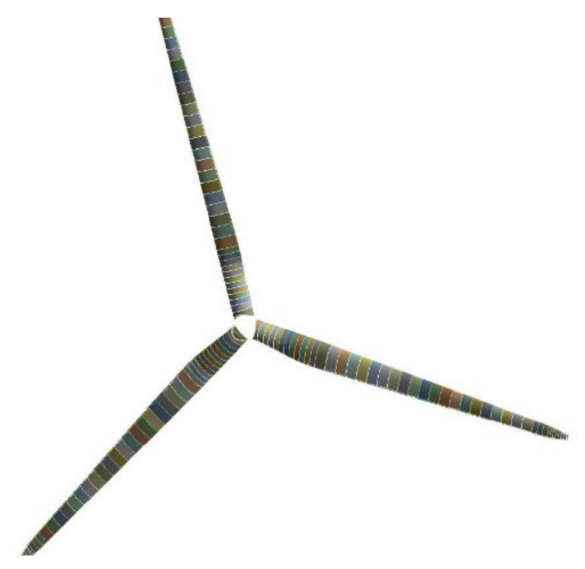

Figure 20. Blade solid geometry.

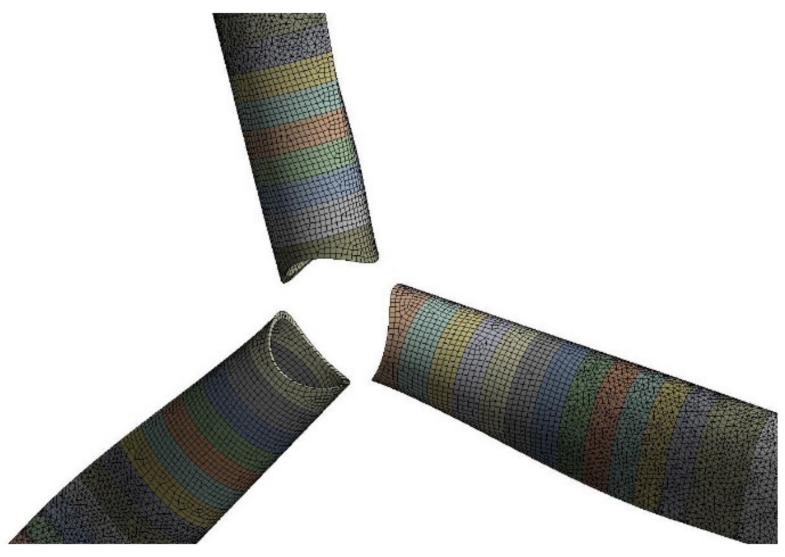

Figure 21. The solid mesh of blades.

Table 3. Blade natural vibration frequency under different vibration modes.

\begin{tabular}{cccc}
\hline Frequency & Mechanical & $\begin{array}{c}\text { FAST (Fatigue, } \\
\text { Aerodynamics, } \\
\text { Structures and } \\
\text { Turbulence) }\end{array}$ & Adams \\
\hline$f_{\text {flapwise1 }}$ & $0.6977 \mathrm{~m}(\triangle=0.21 \%)$ & $0.6991 \mathrm{~m}$ & $0.7001 \mathrm{~m}$ \\
\hline$f_{\text {edgewise1 }}$ & $1.1768 \mathrm{~m}(\triangle=7.82 \%)$ & $1.0914 \mathrm{~m}$ & $1.0867 \mathrm{~m}$ \\
\hline$f_{\text {flapwise2 }}$ & $1.9757 \mathrm{~m}(\triangle=2.19 \%)$ & $2.02 \mathrm{~m}$ & $1.9594 \mathrm{~m}$ \\
\hline
\end{tabular}

When the rotor moved forward, the deformation in the flapwise and edgewise directions were as shown in Figure 22. The blade flapwise was deformed along the $z$-axis direction while the blade edgewise was deformed along the $x$-axis direction. The deformations of the blade tip in the $z$-direction and $x$-direction under the pitch motion were $4.896 \mathrm{~m}$ and $0.693 \mathrm{~m}$, respectively. Under the coupled motion, the deformations of the blade tip in the $z$-direction and $x$-direction were $4.566 \mathrm{~m}$ and $0.636 \mathrm{~m}$, respectively. Lastly, under the yaw motion, the deformations of the tip in the $z$-direction and $x$-direction were $3.628 \mathrm{~m}$ and $0.489 \mathrm{~m}$. Thus, the effect of the pitch motion on the blade deformation was greater than those of the yaw and coupled motions. The reason was that when the rotor moved forward, there were an overlap with the incoming wind speed and an increase in the force in the chord direction. In Section 2, it is shown that the induced velocity under the pitch motion in the chord direction is greater than that in the yawing direction, so the pitch motion causes the maximum displacement. 


\section{Flapwise}

Edgewise
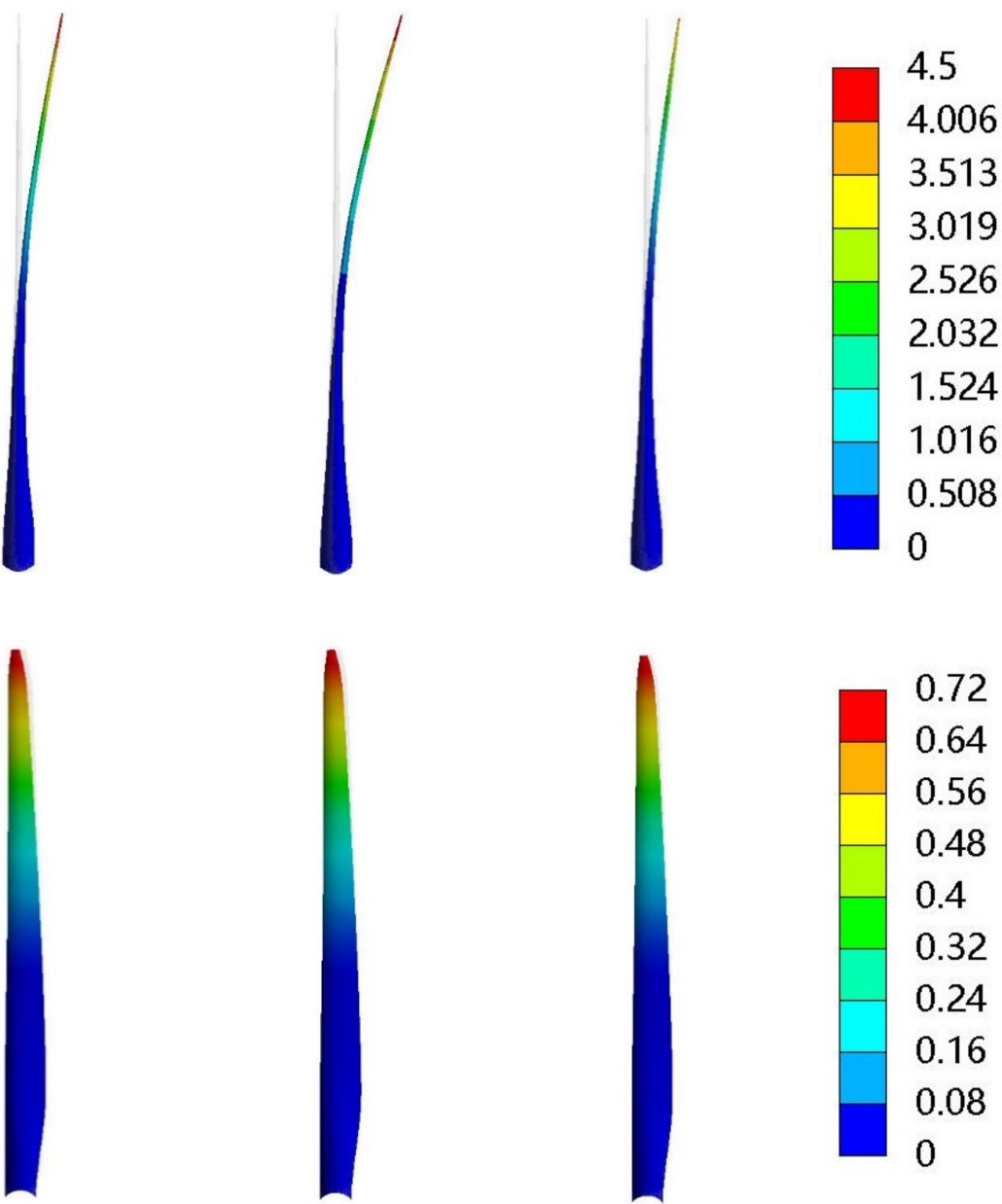

Pitch
Pitch-yaw
Yaw

(unit: $\mathrm{m}$ )

Figure 22. Blade deflection under the forward motion.

When the rotor moved backward, the blade deformations were as shown in Figure 23. The deformations of the blade tip in the $z$-direction and $x$-direction under the pitch motion were $0.515 \mathrm{~m}$ and $0.045 \mathrm{~m}$, respectively. Under the coupled motion, the deformations of the blade tip in the $z$-direction and $x$-direction were $0.674 \mathrm{~m}$ and $0.069 \mathrm{~m}$, respectively. Finally, under the yaw motion, the deformations of the blade tip in the $z$-direction and $x$-direction were $0.927 \mathrm{~m}$ and $0.129 \mathrm{~m}$, respectively. Therefore, backward moving caused smaller blade deformation than forward moving. The reason was that the induced-velocity was opposite to the incoming velocity in the chordwise direction, which made the deformation decreasing with the chordwise velocity. 
Flapwise
Edgewise

Pitch
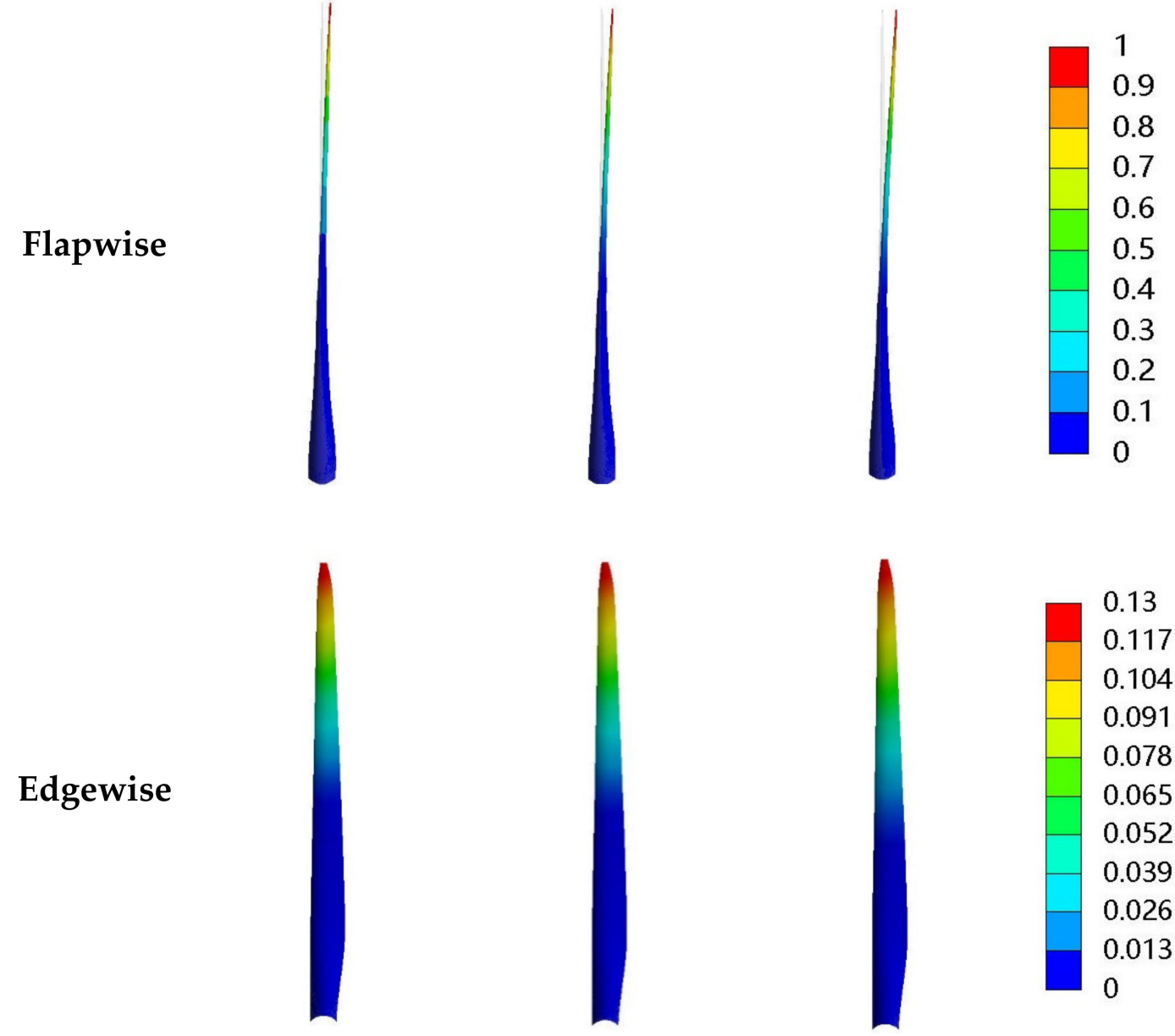

$$
\text { Pitch-yaw }
$$

Yaw

(unit: $\mathrm{m}$ )

Figure 23. Blade deflection under the backward motion.

\section{Conclusions}

This paper presents the results of unsteady numerical simulations investigating the dynamic aerodynamic and structural characteristics of an NREL 5 MW FOWT under the pitch, yaw, and coupled pitch-yaw motions. All of the platform movements are simulated as angular motions, as well as blade rotation. The accuracy of the numerical simulation method is verified. The comparison of the total aerodynamic performance, force coefficients at different spans, and structural dynamic response is also presented.

The conclusions can be drawn as follows:

(1) The increase of platform motion amplitude and frequency can aggravate the fluctuation of wind power, and the influence of pitch motion is greater than that of yaw motion. The platform pitching is the main influencing factor of power fluctuation, which is relative to the platform yawing.

(2) The increase of amplitude and frequency can aggravate the fluctuation range of overall thrust. However, the average thrust values of the pitch, yaw, and coupled motions are consistent, regardless of the frequency value.

(3) The yaw motion has a major impact on the variation in $A O A$. However, the blade normal and tangential forces are mainly affected by the pitch motion. Tangential force coefficient fluctuation of the pitch motion is larger than that of the yaw motion and the coupled motion, which also explains the variation of output power. 
(4) The shell model is used to analyze the structural characteristics of the blade. Both pitch and yaw motions can increase the flapwise and edgewise deformation of the blade. In addition, backward moving caused smaller blade deformation than forward moving, corresponding to the induced velocity variation of two-movement process.

The paper is based on the multi-axis angular motion model and the double-slip mesh technique. This study gives the motion law of FOWT, analyzing the overall and local aerodynamic performance, aerodynamic and structural load, which provides a theoretical basis for the construction, operation, and maintenance of the FOWT. In the future, the aerodynamic performance of FOWTs can be studied under shear, turbulent, or gusty wind, in order to simulate the real offshore wind conditions more accurately.

Author Contributions: Conceptualization, Z.C. and X.W.; methodology, X.W.; software, Z.C. and X.W.; validation, Z.C. and X.W.; formal analysis, Z.C.; investigation, Z.C.; resources, X.W.; data curation, Z.C.; writing —original draft preparation, Z.C.; writing—review and editing, X.W.; visualization, Z.C.; supervision, S.K.; project administration, S.K.; funding acquisition, X.W. and S.K. All authors have read and agreed to the published version of the manuscript.

Funding: The research was supported by National Natural Science Foundation of China (51876063, 51576065).

Institutional Review Board Statement: Not applicable.

Informed Consent Statement: Not applicable.

Data Availability Statement: Not applicable.

Conflicts of Interest: The authors declare no conflict of interest.

\section{References}

1. Zhong, W.; An, H.; Shen, L.; Tao, D.; Wei, F.; Xiangyun, G.; Di, D. Global pattern of the international fossil fuel trade: The evolution of communities. Energy 2017, 123, 260-270. [CrossRef]

2. GWEC. Global Wind Report 2018; GWEC: Brussels, Belgium, 2019.

3. International Energy Agency. Key World Energy Statistics; IAE: Paris, France, 2017.

4. Deng, X.; Ge, J. Global wind power development leads to high demand for Neodymium praseodymium (NdPr): A scenario analysis based on market and technology development from 2019 to 2040. J. Cleaner Prod. 2020, 277. [CrossRef]

5. International Energy Agency. World Energy Outlook 2016 Executive Outlook; IAE: Paris, France, 2016.

6. British Petroleum. BP Energy Outlook to 2035; 2016 Edition; British Petroleum: London, UK, 2016.

7. Pedersen, E.; van den Berg, F.; Bakker, R.; Bouma, J. Response to noise from modern wind farms in The Netherlands. Acoust. Soc. Am. 2009, 126, 634-643. [CrossRef] [PubMed]

8. Pedersen, E.; Persson Waye, K. Perception and annoyance due to wind turbine noise-A dose-response relationship. Acoust. Soc. Am. 2004, 116, 3460-3470. [CrossRef] [PubMed]

9. Van den Berg, F. Criteria for wind farm noise: Lmax and Lden. Acoust. Soc. Am. 2008, 123, 3536. [CrossRef]

10. Wang, X.F.; Zeng, X.W.; Li, J.L. A review on recent advancements of substructures for offshore wind turbines. Energy Conv. Manag. 2018, 158, 103-119. [CrossRef]

11. Katsaprakakis, D.A. A review of the environmental and human impacts from wind parks. A case study for the prefecture of Lasithi, Crete. Renew. Sustain. Energy Rev. 2012, 16, 2850-2863. [CrossRef]

12. Shen, X.; Hu, P.; Jinge, C.; Zhu, X.; Du, Z. The unsteady aerodynamics of floating wind turbine under platform pitch motion. Proceed. Instit. Mechan. Eng. Part A J. Power Energy 2018. [CrossRef]

13. Chen, Z.; Wang, X.; Guo, Y.; Kang, S. Numerical analysis of unsteady aerodynamic performance of floating offshore wind turbine under platform surge and pitch motions. Renew. Energy 2021, 163, 1849-1870. [CrossRef]

14. Bae, Y.H.; Kim, M.H.; Im, S.W. Aero-elastic-control-floater-mooring coupled dynamic analysis of floating offshore wind turbines in Maximum Operation and Survival Conditions. In Proceedings of the ASME 2012 31st International Conference on Ocean, Offshore and Arctic Engineering, Rio de Janeiro, Brazil, 1-6 July 2012.

15. Tran, T.T.; Kim, D. The platform pitching motion of floating offshore wind turbine: A preliminary unsteady aerodynamic analysis. J. Wind Eng. Ind. Aerodyn. 2015, 142, 65-81. [CrossRef]

16. Qiu, Y.X.; Wang, X.D.; Kang, S.; Ming, Z.; Jun-Yu, L. Predictions of unsteady HAWT aerodynamics in yawing and pitching using the free vortex method. Renew. Energy 2014, 70, 93-106. [CrossRef]

17. Wen, B.; Dong, X.; Tian, X.; Zhike, P.; Wenming, Z.; Kexiang, W. The power performance of an offshore floating wind turbine in platform pitching motion. Energy 2018, 154, 508-521. [CrossRef] 
18. Wen, B.R.; Tian, X.L.; Dong, X.J.; Peng, Z.K.; Zhang, W.M.; Wei, K.X. A numerical study on the angle of attack to the blade of a horizontal-axis offshore floating wind turbine under static and dynamic yawed conditions. Energy 2019, 168, 1138-1156. [CrossRef]

19. Lee, H.; Lee, D.-J. Effects of platform motions on aerodynamic performance and unsteady wake evolution of a floating offshore wind turbine. Renew. Energy 2019, 143, 9-23. [CrossRef]

20. Hu, H.; Khosravi, M.; Sarkar, P. An experimental investigation on the aeromechanic performance and wake characteristics of a wind turbine model subjected to pitch motions. In Proceedings of the 34th Wind Energy Symposium, San Diego, CA, USA, 4-8 January 2016; p. 1997.

21. Bayati, I.; Belloli, M.; Bernini, L.; Zasso, A. Wind tunnel wake measurements of floating offshore wind turbines. Energy Proc. 2017, 137, 214-222. [CrossRef]

22. Bayati, I.; Bernini, L.; Zanotti, A.; Zasso, A. Experimental investigation of the unsteady aerodynamics of FOWT through PIV and hot-wire wake measurements. In Proceedings of the Science of Making Torque Conference, Milano, Italy, 20-22 June 2018.

23. Wu, J.; Ding, J.H.; He, Y.P.; Zhao, Y.S. Study on unsteady aerodynamic performance of floating offshore wind turbine by CFD method. In Proceedings of the International Ocean and Polar Engineering Conference, Kona, HI, USA, 21-26 June 2015.

24. Liu, Y.; Xiao, Q.; Incecik, A.; Peyrard, C. Aeroelastic analysis of a floating offshore wind turbine in platform-induced surge motion using a fully coupled CFD-MBD method. Wind Energy 2018, 22, 1-20. [CrossRef]

25. Tran, T.-T.; Kim, D.-H.; Song, J. Computational fluid dynamic analysis of a floating offshore wind turbine experiencing platform pitching motion. Energies 2014, 7, 5011-5026. [CrossRef]

26. Tran, T.-T.; Kim, D.-H. The aerodynamic interference effects of a floating offshore wind turbine experiencing platform pitching and yawing motions. J. Mechan. Sci. Technol. 2015, 29, 549-561. [CrossRef]

27. Wang, X.D.; Ye, Z.L.; Kang, S.; Hu, H. Investigations on unsteady aerodynamic characteristics of a horizontal-axis wind turbine during dynamic yaw process. Energies 2019, 12, 3124. [CrossRef]

28. Wang, X.; Liu, Y.; Wang, L.; Ding, L.; Hu, H. Numerical study of nacelle wind speed characteristics of a horizontal axis wind turbine under time-varying flow. Energies 2019, 12, 3993. [CrossRef] 